\title{
Increase in Mitochondrial Biogenesis in Neuronal Cells by RNS60, a Physically-Modified Saline, via Phosphatidylinositol 3-Kinase-Mediated Upregulation of PGC1a
}

\author{
Goutam Chandra ${ }^{1}$ - Madhuchhanda Kundu ${ }^{1}$. Suresh B. Rangasamy ${ }^{1}$ • \\ Sridevi Dasarathy ${ }^{1} \cdot$ Supurna Ghosh $^{2} \cdot$ Richard Watson $^{2} \cdot$ Kalipada Pahan ${ }^{1}$
}

Received: 31 October 2017 / Accepted: 10 November 2017 / Published online: 29 November 2017

(C) The Author(s) 2017. This article is an open access publication

\begin{abstract}
This study highlights a novel approach to upregulate mitochondrial biogenesis in neuronal cells. RNS60 is a $0.9 \%$ saline solution containing oxygenated nanobubbles that is generated by subjecting normal saline to Taylor-Couette-Poiseuille (TCP) flow under elevated oxygen pressure. RNS60, but not NS (normal saline), PNS60 (saline containing a comparable level of oxygen without the TCP modification), or RNS10.3 (TCP-modified normal saline without excess oxygen), increased the expression of Nrfl, Tfam, Mcu, and Tom20 (genes associated with mitochondrial biogenesis) and upregulated mitochondrial biogenesis in MN9D dopaminergic neuronal cells. Similarly RNS60 also increased mitochondrial biogenesis in primary dopaminergic neurons and in the nigra of MPTP-intoxicated mice. However, RNS60 had no effect on lysosomal biogenesis. Interestingly, we found that RNS60 upregulated PGC1 $\alpha$ and siRNA knockdown of PGC1 $\alpha$ abrogated the ability of RNS60 to increase mitochondrial biogenesis. Furthermore, we delineated that RNS60 increased the transcription of Pgcla via type IA phosphatidylinositol (PI) 3-kinase-mediated activation of cAMP-response element-binding protein (CREB). Accordingly, knockdown of the PI3K - CREB pathway suppressed RNS60-mediated mitochondrial biogenesis. These results describe a novel property of RNS60 of enhancing mitochondrial biogenesis via PI 3-kinase-CREB-mediated up-regulation of PGC1 $\alpha$, which may be of therapeutic benefit in different neurodegenerative disorders.
\end{abstract}

Keywords Mitochondrial biogenesis $\cdot$ Neurons $\cdot$ PGC1 $\alpha \cdot$ Modified saline $\cdot$ Nanobubbles $\cdot$ PI3 kinase

\section{Introduction}

Mitochondrion is the most important organelle in terms of energy metabolism and neurons in the central nervous system (CNS) have an intense demand for mitochondrial function. Recent studies indicate alteration in mitochondrial homeostasis in the CNS of patients with different neurodegenerative diseases. While complex I activity is reduced in I in Parkinson's disease (PD), activities of cytochrome oxidase and multiple electron transport chain (ETC) enzymes are decreased in Alzheimer's disease (AD) and Huntington's

Goutam Chandra and Madhuchhanda Kundu contributed equally to this work.

Kalipada Pahan

Kalipada_Pahan@rush.edu

1 Department of Neurological Sciences, Rush University Medical Center, 1735 West Harrison St, Suite Cohn 310, Chicago,

IL 60612, USA

2 Revalesio Corporation, 1200 East D Street, Tacoma, WA 98421, USA disease, respectively (Mizuno et al. 1995; Hirai et al. 2001; Baloyannis 2006; Surmeier and Sulzer 2013; Guedes-Dias et al. 2015). Mitochondrial dysfunction has also been reported in amyotrophic lateral sclerosis and progressive supranuclear palsy (Albers and Beal 2002; Tafuri et al. 2015). Leber's hereditary optic neuropathy, a focal degeneration of the optic nerves, is known to arise due to mutations in mtDNAencoded complex I genes and is associated with complex I dysfunction (Carelli et al. 2004). Mitochondrial dysfunction also participates in the pathogenesis of epilepsy (Zsurka and Kunz 2015). Since different neurodegenerative diseases are associated with mitochondrial malfunction, it is important to focus on developing methods to repair and restore mitochondria. Enhancing mitochondrial biogenesis may have therapeutic importance for different neurodegenerative diseases.

The diverse functionality of mitochondria requires a very complex and coordinated regulation of its activity with transcription factors NRFs/PPARs/ERRs that activate target genes encoding enzymes of fatty acid metabolism, oxidative phosphorylation and anti-oxidant defense (Scarpulla 2002; Fan and Evans 2015). By co- 
activating and regulating the expression of this network, $\mathrm{PGC} 1 \alpha$, the master regulator of mitochondrial biogenesis, directly links external physiological stimuli to the regulation of mitochondrial biogenesis and function (Wu and Boss 2007; Wareski et al. 2009). However, mechanisms by which $\mathrm{PGC} 1 \alpha$ is activated and mitochondrial biogenesis is upregulated are poorly understood. RNS60 is physically modified saline that contains no active pharmaceutical ingredients. It is generated by subjecting normal saline to Taylor-Couette-Poiseuille (TCP) flow under elevated oxygen pressure (Khasnavis et al. 2012; Mondal et al. 2012; Roy et al. 2014). Recently, we have demonstrated that RNS60 exerts antiinflammatory effects in glial cells via suppression of nuclear factor kappa B (NF- $\mathrm{KB}$ ) activation (Khasnavis et al. 2012). RNS60 also protects dopaminergic neurons in MPTP mouse model of PD (Khasnavis et al. 2014) and hippocampal neurons in a mouse model of AD (Modi et al. 2015). Here, we delineate that RNS60 stimulated mitochondrial biogenesis and increased the expression of Nrfl, Tfam, Mcu, and Tom20 (genes associated with mitochondrial biogenesis) in dopaminergic neuronal cells. However, RNS60 did not have any such stimulatory effect on lysosomal biogenesis. Furthermore, we demonstrate that RNS60 induced the activation of type IA $\mathrm{PI} 3 \mathrm{~K}$ and that RNS60 increased mitochondrial biogenesis via type IA PI3K-CREB-mediated upregulation of PGC1 $\alpha$. Finally, MPTP intoxication reduced the expression of PGC $1 \alpha$ and decreased mitochondrial biogenesis, which were increased by RNS60 treatment. Our studies suggest that this physically-modified saline may be of therapeutic value in the treatment of PD and other neurodegenerative disorders in which mitochondrial abnormality plays an important role.

\section{Methods}

Cells MN9D cells were obtained from Dr. A. Heller (University of Chicago, Chicago, IL, USA). Cells were maintained in Dulbecco's modified Eagle's medium (Thermo Fisher Scientific, Waltham, MA) supplemented with $10 \%$ $(v / v)$ heat-inactivated fetal bovine serum, $3.7 \mathrm{~g} / \mathrm{L} \mathrm{NaHCO}_{3}$, $50 \mathrm{U} / \mathrm{mL}$ penicillin, and $50 \mu \mathrm{g} / \mathrm{mL}$ streptomycin in an incubator with an atmosphere of $7 \% \mathrm{CO}_{2}$ at $37^{\circ} \mathrm{C}$. These cells express abundant tyrosine hydroxylase, synthesize dopamine (DA) and also quantitatively release DA (Choi et al. 1991; Perez et al. 2002; Dong et al. 2008).

Reagents Hank's balanced salt solution (HBSS) and 0.05\% trypsin were purchased from Mediatech (Washington, DC). Fetal bovine serum (FBS) was obtained from Atlas Biologicals (Fort Collins, CO). Antibiotic-antimycotic mixture was obtained from Sigma-Aldrich (St. Louis, MO).
Isolation of Mouse Primary Dopaminergic Neurons Nigra was dissected as a thin slice of ventral mesencephalon tissue from E12.5 to 14 days old fetus and homogenized with $1 \mathrm{ml}$ of trypsin for $5 \mathrm{~min}$ at $37^{\circ} \mathrm{C}$ followed by neutralization of trypsin as described earlier (Roy and Pahan 2013). The single cell suspension of nigral tissue was plated in the poly-D-lysine pre-coated $75 \mathrm{~mm}$ flask and was allowed to differentiate fully for 9-10 days before treatment (Roy and Pahan 2013).

Animals and MPTP Intoxication Six- to eight-weeks old C57BL/6 mice were purchased from Harlan, Indianapolis, IN. Animal maintenance and experiments were in accordance with National Institutes of Health guidelines and were approved by the Institutional Animal Care and Use committee of the Rush University Medical Center, Chicago, IL. For acute MPTP intoxication, mice received four intraperitoneal (i.p.) injections of MPTP-HCl $(18 \mathrm{mg} / \mathrm{kg}$ of free base; Sigma Chemical Co., St. Louis, MO) in saline at 2-h intervals (Ghosh et al. 2007; Ghosh et al. 2009; Roy et al. 2012). Control animals received only saline.

Preparation of RNS60 RNS60 was generated at Revalesio (Tacoma, WA) as described before (Khasnavis et al. 2012; Mondal et al. 2012). Briefly, sodium chloride (0.9\%) for irrigation, USP pH 5.6 (4.5-7.0, Hospira), was processed at $4^{\circ} \mathrm{C}$ using Taylor-Couette-Poiseuille (TCP) flow and a flow rate of $32 \mathrm{~mL} / \mathrm{s}$ under $1 \mathrm{~atm}$ of oxygen back-pressure $(7.8 \mathrm{~mL} / \mathrm{s}$ gas flow rate), while maintaining a rotor speed of $3450 \mathrm{rpm}$. Chemically, RNS60 contains water, sodium chloride, 50-60 ppm oxygen, but no active pharmaceutical ingredients. The following controls for RNS60 were also used in this study:

a) NS, normal saline from the same manufacturing batch. This saline contacted the same device surfaces as RNS60 and was bottled in the same way.

b) RNS10.3, a saline that was processed with TCP flow in the absence of any excess oxygen.

c) PNS60, saline with the same oxygen content as RNS60 $(55 \pm 5 \mathrm{ppm})$ that was prepared inside of the same device but was not processed with TCP flow.

Careful analysis demonstrated that all four fluids were chemically identical (Khasnavis et al. 2012). Liquid chromatography quadrupole time-of-flight mass spectrometric analysis also showed no difference between RNS60 and other control solutions (Khasnavis et al. 2012). Atomic force microscopy showed that RNS60 displayed different surface nanobubble nucleation characteristics relative to that of control saline solutions (Khasnavis et al. 2012) even when they contained similar levels of dissolved oxygen.

RNS60/NS Treatment of Mice Mice were treated with RNS60 or NS $(300 \mu 1 /$ mouse/d) via intraperitoneal (i.p.) injection from $3 \mathrm{~h}$ after the last injection of MPTP for 7 days as described (Khasnavis et al. 2014). 
Antibodies Rabbit polyclonal anti-PGC $1 \alpha$, mouse monoclonal anti-Nrfl and rabbit polyclonal anti-TFAM antibodies were obtained from Abcam (Cambridge, MA). Anti-tyrosine hydroxylase (TH), anti-p300 and anti-NeuN antibodies were obtained Millipore (Billerica, MA). Rabbit monoclonal antibodies against phospho-CREB, total CREB and CREBbinding protein (CBP) were purchased from Cell Signaling (Beverly, MA). Cy2- and Cy5-conjugated antibodies were obtained from Jackson Immuno Research Laboratories (West Grove, PA).

Assay of PI3K Activation Upon activation, PI3K subunits translocate to the plasma membrane. Therefore, after different time of treatment with RNS60 and NS, subunits of PI3K were monitored in plasma membrane. Briefly, cells were dissociated in $100 \mathrm{mM}$ sodium bicarbonate buffer $(\mathrm{pH} 11.5)$ and spun in an ultracentrifuge at 40,000 rpm for $1 \mathrm{~h}$ at $4^{\circ} \mathrm{C}$. The resultant supernatant was aspirated and the pellet was immersed in double-distilled water and SDS and stored at $-80^{\circ} \mathrm{C}$ overnight. The following day, the pellet was resuspended by repeated grinding and boiling, and processed for Western blot using antibodies against $\mathrm{p} 110 \alpha, \mathrm{p} 110 \beta$ and $\mathrm{p} 110 \gamma$ (Khasnavis et al. 2014).

Western Blot Analysis Western blotting was conducted as described earlier (Corbett et al.; Saha et al. 2006) with modifications. Briefly, cells were scraped in 1X RIPA buffer, protein was measured using Bradford reagent and sodium dodecyl sulfate (SDS) buffer was added and electrophoresed on NuPAGE® Novex ${ }^{\circledR} 4-12 \%$ Bis-Tris gels (Life Technologies, Grand Island, NY) and proteins transferred onto a nitrocellulose membrane (Bio-Rad) using the ThermoPierce Fast Semi-Dry Blotter. The membrane was then washed for $15 \mathrm{~min}$ in TBS plus Tween 20 (TBST) and blocked for $1 \mathrm{~h}$ in TBST containing BSA. Next, membranes were incubated overnight at $4^{\circ} \mathrm{C}$ under shaking conditions with primary antibodies. The next day, membranes were washed in TBST for $1 \mathrm{~h}$, incubated in secondary antibodies against for $1 \mathrm{~h}$ at room temperature, washed for one more hour and visualized under the Odyssey® Infrared Imaging System (LiCOR, Lincoln, NE).

Semi-Quantitative RT-PCR Analysis Total RNA was isolated from ventral midbrain using Ultraspec-II RNA reagent (Biotecx Laboratories, Inc., Houston, TX) following the manufacturer's protocol. To remove any contaminating genomic DNA, total RNA was digested with DNase. RT-PCR was carried out as described earlier (Jana et al. 2007; Brahmachari et al. 2009; Ghosh et al. 2009) using a RT-PCR kit (Clontech, Mountain View, CA) and the following primers:

NRF1: Sense: 5'-CTTCATGGAGGAGCACGGAG-3'

Antisense: 5'-ACTGTGCTCAGTGTACGTGG-3'
TFAM: Sense: 5'-CAGTAGCCTTGTGGGCTTTC-3'

Antisense: 5'-CTGCCATGTGTTCTCCTGGG-3'

TOMM20: Sense: 5'-GTGCCATCTTGACGGGAGAT$3^{\prime}$

Antisense: 5'-GCACTGATGCAAGTGAGCTG-3'

MCU: Sense: 5'-GCCACCAAAGAGAGACCTCC-3'

Antisense: 5'-GCTCAATGCACAGTGTGGTG-3'

PGC1 $\alpha$ : Sense: 5'-TCTCAGTAAGGGGCTGGTTG-3'

Antisense: 5'-TTCCGATTGGTCGCTACACC-3'

TFEB: Sense: 5'-AAC AAA GGC ACC ATC CTC AA $3^{\prime}$

Antisense: 5'-CAG CTC GGC CAT ATT CAC AC-3'

LAMP2: Sense: 5'-GGT GCT GGT CTT TCA GGC TTG ATT-3'

Antisense: 5'- ACC ACC CAA TCT AAG AGC AGG ACT-3'

GAPDH: Sense: 5'-GGTGAAGGTCGGTGTGAACG$3^{\prime}$

Antisense: 5'-TTGGCTCCACCCTTCAAGTG-3'.

Real-Time PCR Analysis The mRNA quantification was performed using the ABI-Prism7700 sequence detection system (Applied Biosystems, Foster City, CA) using SYBR Select master mix (Applied Biosystems). The mRNA expression of the targeted genes was normalized to the level of Gapdh mRNA and data was processed by the ABI Sequence Detection System 1.6 software as described earlier (Jana et al. 2007; Brahmachari et al. 2009; Ghosh et al. 2009; Khasnavis and Pahan 2012).

Quantification of Mitochondrial DNA (mtDNA) Total DNA was isolated from MN9D cells using QIAamp DNA mini kit (Qiagen). The mtDNA content relative to nuclear DNA was assessed by real-time PCR using the ABI7500 (Applied Biosystems).

Primers of the mtDNA encoded Coxl gene:

Forward: 5'-TGCTAGCCGCAGCATTAC-3'

Reverse: 5'-GGGTGCCCAAAGAATCAGAAC-3'

Primers of the single copy nuclear gene Ndufv1:

Forward: 5'-CTTCCCCACTGGCCTCAAG-3' 
Reverse: 5'-CCAAAACCCAGTGATCCAGC-3'.

Relative mtDNA was determined using $\Delta \mathrm{C}_{\mathrm{T}}$ method.

\section{Monitoring Mitochondrial Membrane Potential}

By Microplate Reader Briefly, cells were plated in 96-well microplates and treated with RNS60 and NS for $18 \mathrm{~h}$ under serum-free condition. Cells were incubated with $500 \mathrm{nM}$ TMRE for $30 \mathrm{~min}$ at $37^{\circ} \mathrm{C}$. Media was replaced with $100 \mathrm{uL}$ PBS $/ 0.2 \%$ BSA. Plate was read on a PerkinElmer fluorescence plate reader with Ex/Em 549/575 nm range.

By FACS Analysis Briefly, $10^{5}$ cells were plated and treated with RNS60 and NS for $18 \mathrm{~h}$ under serum-free condition. Cells were incubated with $100 \mathrm{nM}$ TMRE for $30 \mathrm{~min}$ at $37^{\circ} \mathrm{C}$. Media was replaced with PBS $/ 0.2 \%$ BSA for flow cytometry analysis. TMRE signal was detected in PE-FL2 channel in a BD FACSCanto II Flow Cytometer.

Chromatin Immunoprecipitation Assay ChIP assays were performed as described earlier by us with a few modifications (Roy et al. 2013; Roy et al. 2015). Briefly, after treatment, cells were fixed with formaldehyde $(1.42 \%$ final volume) and quenched with $125 \mathrm{mM}$ glycine. The cells were then pelleted and lysed in IP buffer containing $150 \mathrm{mM} \mathrm{NaCl}, 50 \mathrm{mM}$ Tris-HCl (pH 7.5), $5 \mathrm{mM}$ EDTA, NP-40 ( $0.5 \% \mathrm{vol} / \mathrm{vol})$, Triton X-100 (1.0\% vol/vol). For $500 \mathrm{ml}$, add $4.383 \mathrm{~g} \mathrm{NaCl}, 25 \mathrm{ml}$ of $100 \mathrm{mM}$ EDTA (pH 8.0), $25 \mathrm{ml}$ of $1 \mathrm{M}$ Tris- $\mathrm{HCl}(\mathrm{pH} 7.5$ ), $25 \mathrm{ml}$ of $10 \%$ $(v / v)$ NP-40 and $50 \mathrm{ml}$ of $10 \%(v / v)$ Triton X-100 containing the following inhibitors; $10 \mu \mathrm{g} / \mathrm{ml}$ leupeptin, $0.5 \mathrm{mM}$ phenylmethlysulfonyl fluoride (PMSF), $30 \mathrm{mM}$ pnitrophenyl phosphate, $10 \mathrm{mM} \mathrm{NaF}, 0.1 \mathrm{mM} \mathrm{Na}_{3} \mathrm{VO}_{4}$, $0.1 \mathrm{mM} \mathrm{Na}_{2} \mathrm{MoO}_{4}$ and $10 \mathrm{mM} \beta$-glycerophosphate. After one wash with $1.0 \mathrm{ml}$ IP buffer, the pellet was resuspended in $1 \mathrm{ml} \mathrm{IP} \mathrm{buffer} \mathrm{(containing} \mathrm{all} \mathrm{inhibitors)} \mathrm{and} \mathrm{sonicated}$ and sheared chromatin was split into two fractions (one to be used as Input). The remaining fraction was incubated overnight under rotation at $4^{\circ} \mathrm{C}$ with 3-4 $\mu \mathrm{g}$ of anti-CREB, anti-CBP or anti-p300 antibodies followed by incubation with Protein G-Agarose (Santa Cruz) for $2 \mathrm{~h}$ at $4^{\circ} \mathrm{C}$ under rotation. Normal IgG was also run as control. Beads were then washed five times with cold IP buffer and a total of $100 \mu \mathrm{l}$ of $10 \%$ Chelex $\left(10 \mathrm{~g} / 100 \mathrm{ml} \mathrm{H}_{2} \mathrm{O}\right)$ was added directly to the washed protein $\mathrm{G}$ beads and vortexed. After 10 min boiling, the Chelex/protein $\mathrm{G}$ bead suspension was allowed to cool to room temperature. Proteinase K $(100 \mu \mathrm{g} / \mathrm{ml})$ was then added and beads were incubated for $30 \mathrm{~min}$ at $55^{\circ} \mathrm{C}$ while shaking, followed by another round of boiling for $10 \mathrm{~min}$. The suspension was centrifuged and supernatant collected. The Chelex/protein $G$ beads fraction was vortexed with another $100 \mu \mathrm{l}$ water, centrifuged again, and the first and the second supernatants were combined. Eluate was used directly as a template in
PCR. The following primers were used to amplify fragments flanking CRE in the mouse Pgcl $\alpha$ promoter:

\section{Sense: 5'-GCGTTACTTCACTGAGGCAG-3' Antisense: 5'-CAGCCTCCCTTCTCCTGTGC-3'}

The PCRs were repeated by using varying cycle numbers and different amounts of templates to ensure that results were in the linear range of PCR. Quantitative real-time PCR was also performed using the same primers and SYBR select MasterMix. Data were normalized to input and nonspecific $\mathrm{IgG}$, and fold increase versus control was calculated.

Immunostaining of Cells Immunocytochemistry was performed as described earlier (Khasnavis and Pahan). Briefly, after treatment, cells were fixed with chilled Methanol (Fisher Scientific, Waltham, MA) overnight, followed by two brief rinses with filtered PBS. Samples were blocked with $2 \%$ BSA (Fisher Scientific) in PBS containing Tween 20 (Sigma) and Triton X-100 (Sigma) for $30 \mathrm{~min}$ and incubated at room temperature under shaking conditions for $2 \mathrm{~h}$ in PBS containing the following anti-mouse primary antibodies: TFAM (1:500), PGC1 $\alpha$ (1:300), Nrf1 (1:300), and NeuN (1:500). After four 15 min washes in filtered PBS, the slides were further incubated with Cy2 or Cy5-labeled secondary antibodies (all 1:200; Jackson ImmunoResearch, West Grove, PA) for $1 \mathrm{~h}$ under similar shaking conditions. Following four 15 min washes with filtered PBS, cells were incubated for 4-5 min with 4', 6-diamidino-2-phenylindole (DAPI, 1:10,000; Sigma). The samples were run in an EtOH and Xylene (Fisher) gradient, mounted, and observed under Olympus BX41 fluorescence microscope.

Immunostaining of Tissue Sections After treatment, mice were sacrificed and their brains fixed, embedded, and processed. Sections were made from ventral midbrain regions and for immunofluorescence staining on fresh frozen sections, antimouse TFAM (1:300), anti-mouse Nrf1 (1:200), anti-mouse PGC1 $\alpha$ (1:200), and anti-mouse NeuN (1:500) were used (Ghosh et al. 2015). The samples were mounted and observed under Olympus BX41 fluorescence microscope (Dasgupta et al. 2004).

Electron Microscopy and Counting of Mitochondria and Lysosomes After treatment, cells were fixed with paraformaldehyde (2\%) and glutaraldehyde (2.5\%) mixture. After primary fixation, samples were prepared in the Electron Microscopy core facility of the University of Illinois at Chicago Research Resources Center. To stabilize cell components, samples were treated with $1 \%$ osmium tetroxide in phosphate buffer. Next, the samples were dehydrated through an increasing concentration of ethanol, passed through propylene oxide and then infiltrated and embedded in a liquid resin. Resin block is then sectioned by ultramicrotomy with $50-70 \mathrm{~nm}$ thickness and collected on metal mesh 'grids' followed by staining these 
grids with electron dense stains before observation in the TEM (JEOL JEM-1220).

Counting analysis was performed using Olympus Microsuite V software with the help of a touch counting module. Before counting cells, the entire image area was calibrated with the help of a rectangular box available in the touch counting panel. Once the area of the image was measured, the touch counting program was applied to count the number of mitochondria or lysosomes using a simple mouse click method. Next, the total number of signals in a given area was normalized by the total area of the image and presented as number of cells per square millimeter unit. Counting was done for 20 fields in each treatment group.

MitoTracker Staining Cells cultured to $70-80 \%$ confluence were subjected to different stimuli under serum-free condition followed by incubation with $75 \mathrm{nM}$ MitoTracker Red (Thermo Fisher Scientific, Waltham, MA) for 45mins. Cells were then washed thoroughly with filtered PBS and mounted on glass slides and viewed under BX41 fluorescence microscope. Cells were visualized with a $100 \times$ objective and MitoTracker signals were counted as described above using Olympus Microsuite V software.

Statistics All values are expressed as means \pm SEM. One-way ANOVA was performed while analyzing dose-dependent effect of RNS60 on mRNA expression of Nrf1, Tfam, Mcu, and Tom 20 in neuronal cells. In other cases, Student's $t$-test was used to compare outcome between two groups (e.g. control vs MPTP, MPTP vs RNS60 etc.).

\section{Results}

RNS60 Increases the Biogenesis of Mitochondria, but Not Lysosomes, in MN9D Dopaminergic Neuronal Cells Increasing mitochondrial biogenesis in neurons may have implications in neurodegenerative disorders. Therefore, we first investigated whether RNS60 containing charge-stabilized nanostructures (Khasnavis et al. 2012; Khasnavis et al. 2014) could upregulate mitochondrial biogenesis in MN9D neuronal cells. Cells treated with RNS60 for $24 \mathrm{~h}$ under serum-free condition were double-labeled with MitoTracker and tyrosine hydroxylase (TH). Although RNS60 did not modulate the level of $\mathrm{TH}$, we found dose-dependent increase in mitochondrial content per cell after RNS60 treatment (Fig. 1a-b). On the other hand, unprocessed NS from the same manufacturing batch had no such stimulatory effect on mitochondrial biogenesis (Fig. 1c). Furthermore, RNS10.3, saline that was processed with TCP flow in the absence of any excess oxygen, and PNS60, saline with same oxygen content as RNS60 $(55 \pm 5 \mathrm{ppm})$ that was prepared inside of the same device but was not processed with TCP flow (Khasnavis et al. 2012), also remained unable to stimulate mitochondrial content in neuronal cells (Fig. 1c). To confirm this finding further, we also monitored mitochondrial DNA. Similar to MitoTracker labeling, RNS60, but not NS, RNS10.3 and PNS60, increased mitochondrial DNA in MN9D neuronal cells (Fig. 1d). These results suggest that stimulation of mitochondrial biogenesis in neuronal cells is specific to RNS60.

Next, we monitored the effect of RNS60 on lysosomal biogenesis to test if RNS60's effects are organelle-specific. Under the same treatment condition as described above, cells were double-labeled with LysoTracker and TH. In contrast to the stimulation of MitoTracker labeling by RNS60, we did not see any increase in LysoTracker staining in RNS60-treated neuronal cells (Fig. 1e-f). To confirm these finding further, we performed electron microscopy (EM) of MN9D neuronal cells after RNS60 and NS treatment. Similar to MitoTracker labeling, RNS60, but not NS, upregulated the number of mitochondria (Fig. 2a-b) and increased mitochondrial diameter (Fig. 2c) and area (Fig. 2d) in MN9D neuronal cells. On the other hand, either RNS60 or NS had no effect on lysosomal biogenesis (Fig. 2a and e). Consistent to increase in mitochondrial number, shape and size, RNS60, but not NS, also increased ATP production (Fig. 2f) and stimulated mitochondrial membrane potential (Fig. 2g-i) in MN9D neuronal cells.

RNS60 Increases the Expression of Genes Associated with Mitochondrial Biogenesis in MN9D Dopaminergic Neuronal Cells Although mitochondria has its own genome, the majority of mitochondrial proteins are encoded in the nucleus. For example, nuclear respiratory factor 1 is linked to the transcriptional control of many mitochondrial genes. Mitochondrial transcription factor A (TFAM), the transcription factor controlling the transcription of mitochondrial genes (Kukat and Larsson 2013), is also transcribed in the nucleus. Therefore, we examined if RNS60 could modulate the expression of genes responsible for mitochondrial biogenesis. It is clearly evident from Fig. 3a that RNS60 dose-dependently increased the mRNA expression of Nrfl and Tfam as well as Tomm 20 and $\mathrm{Mcu}$, genes that encode mitochondrial membrane proteins translocase of outer mitochondrial membrane 20 (TOMM20) and mitochondrial calcium uniporter (MCU), respectively (Schleiff and Turnbull 1998; Petrungaro et al. 2015). These results are also supported by real-time PCR analysis (Fig. 3be). On the other hand, consistent to LysoTracker results, RNS60 was unable to stimulate the expression of Tfeb and Lamp2, genes that are responsible for lysosomal biogenesis, at any of the doses tested (Fig. $3 \mathrm{f}-\mathrm{g}$ ). To understand the specificity, cells were also treated with NS, RNS10.3 and PNS60. Similar to MitoTracker results, NS, RNS10.3 and PNS60 remained unable to stimulate the expression of $\mathrm{Nrfl}$ and Tfam in MN9D neuronal cells (Fig. 3h-j), indicating that the induction of $\mathrm{Nrfl}$ and Tfam is specific for RNS60. As expected, NS, RNS10.3 and PNS60 also did not modulate the expression of Tfeb and Lamp2 (Fig. 3k-1). 


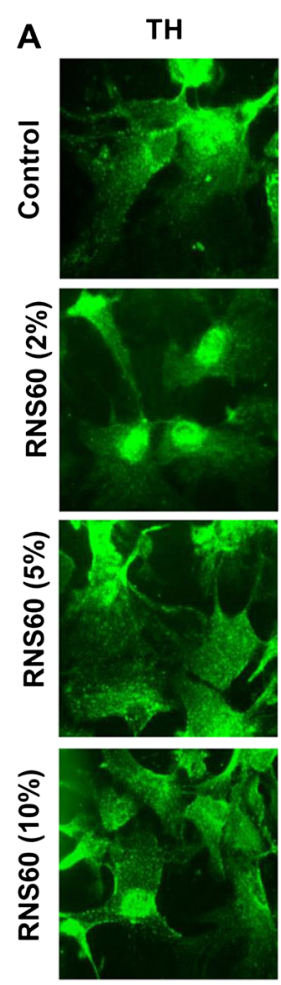

\section{MitoTracker}
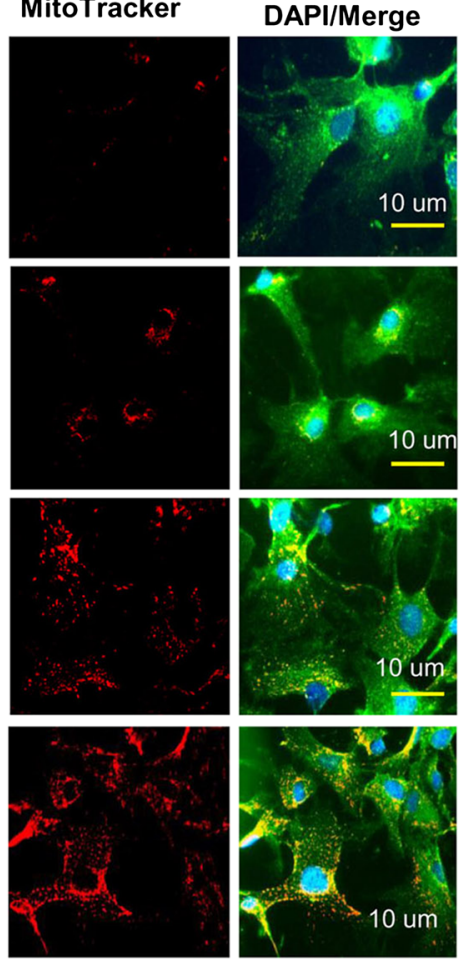

B
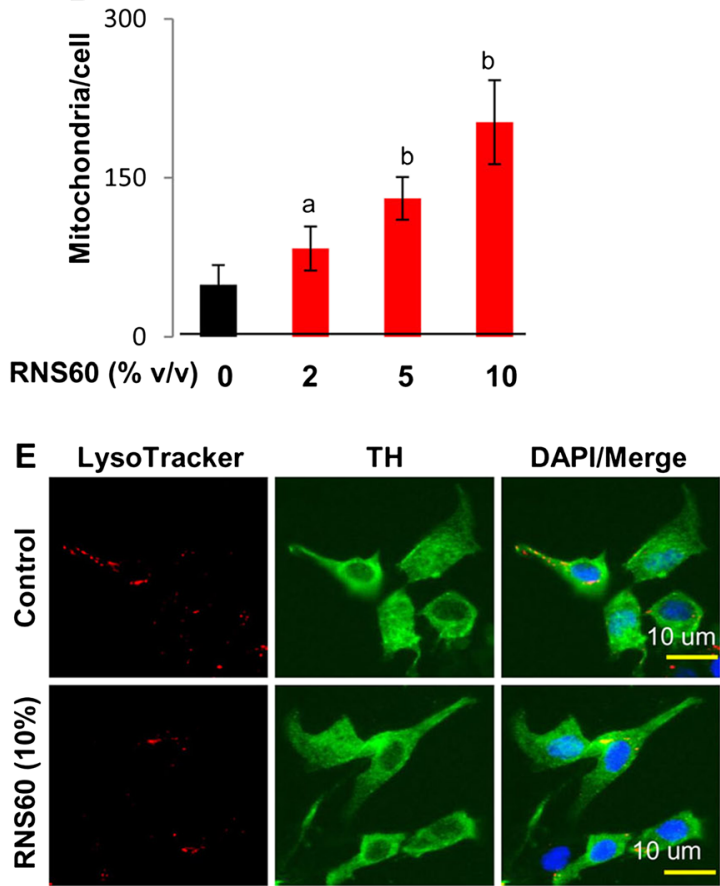

Fig. 1 RNS60 stimulates mitochondrial biogenesis in MN9D mouse neuronal cells. a Cells were treated with different concentrations of RNS60 under serum-free condition for $24 \mathrm{~h}$ followed by MitoTracker labeling and immunostaining with anti-TH antibody. b Number of mitochondria was counted in 25 different cells and expressed as mitochondria per cell. Data are mean \pm SEM of 25 different cells per group. ${ }^{a} p<0.05$ versus control; ${ }^{b} p<0.001$ versus control. c Cells were treated with $10 \%$ $v / v$ RNS60, NS, RNS10.3, or PNS60 under serum-free condition for $24 \mathrm{~h}$
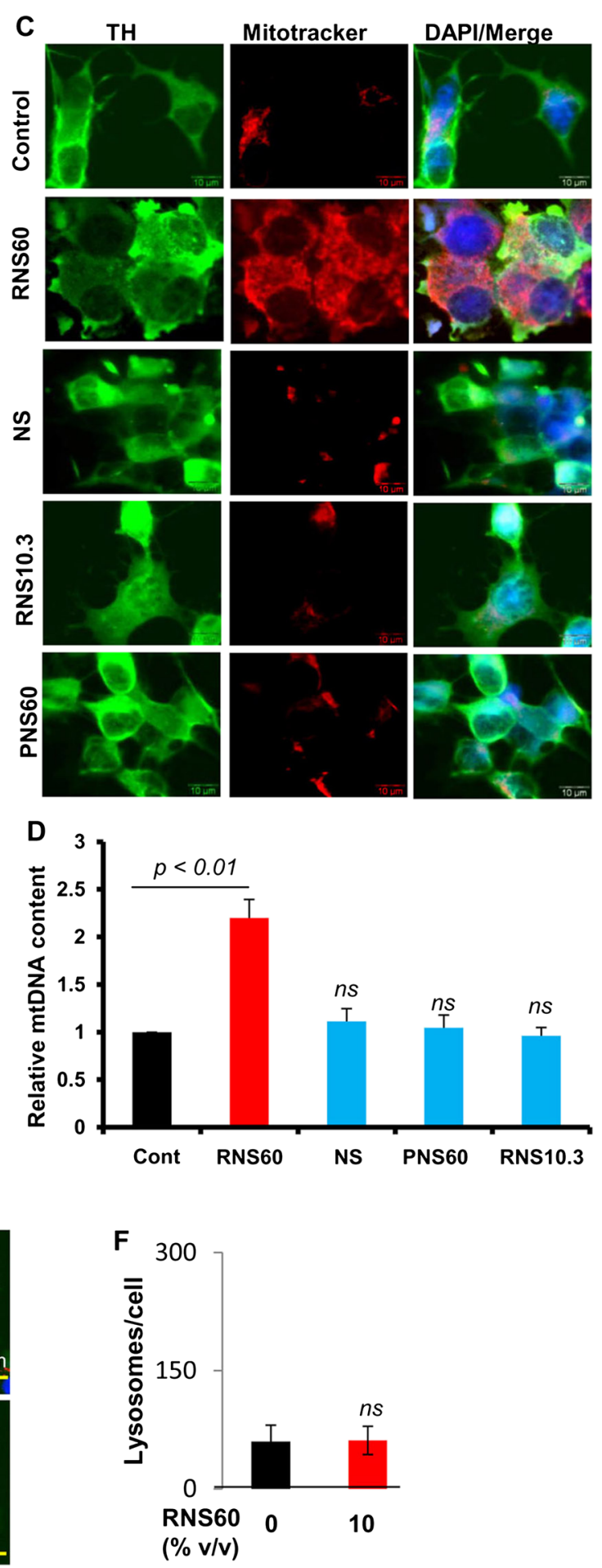

followed by MitoTracker labeling and immunostaining with anti-TH antibody. d Under same treatment condition, mitochondrial DNA content was monitored. e Cells were treated with $10 \% v / v$ RNS60 under serumfree condition for $24 \mathrm{~h}$ followed by LysoTracker labeling and immunostaining with anti-TH antibody. f Number of lysosomes was counted in 25 different cells and expressed as lysosomes per cell. Data are mean \pm SEM of 25 different cells per group 

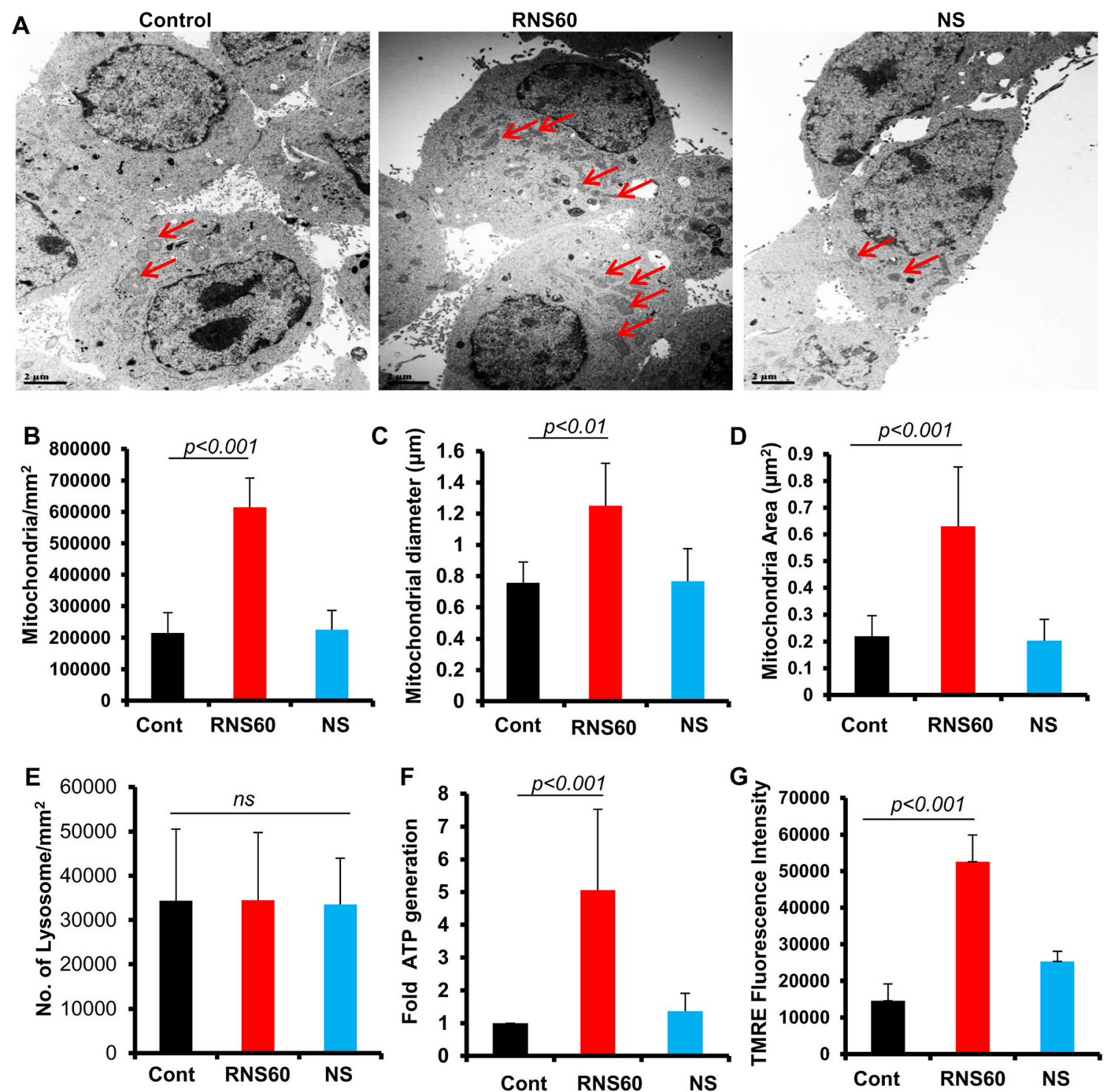

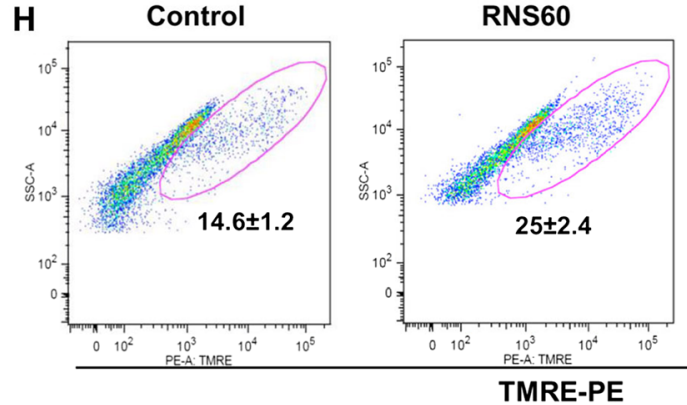

Fig. 2 Electron microscopic detection of mitochondria and determination of mitochondrial membrane potential in RNS60treated MN9D mouse neuronal cells. a Cells were treated with RNS60 $(10 \% v / v)$ and NS $(10 \% v / v)$ under serum-free condition for $24 \mathrm{~h}$ and processed for electron microscopy as described under "Materials and Methods". Number of mitochondria (b) was counted using Olympus Microsuite V software with the help of a touch counting module. Results are mean $\pm \mathrm{SEM}$ of 20 fields. Mean mitochondrial diameter $\max (\mathbf{c})$ and area (d) was determined by measuring at least 80 individual mitochondria from images of multiple cells using Olympus
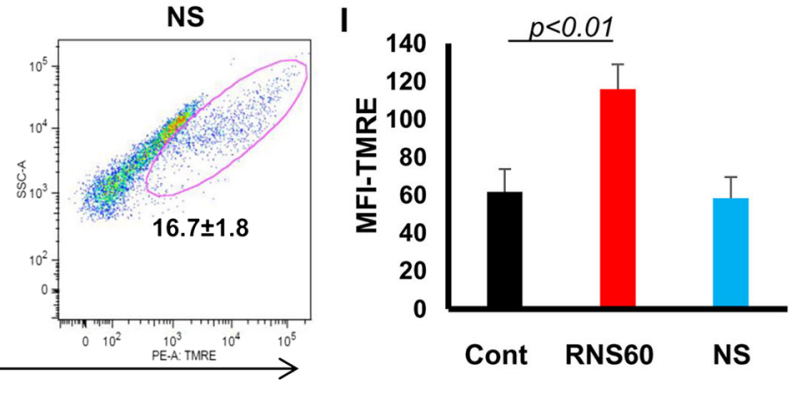

Microsuite V software. Number of lysosomes (e) was counted using Olympus Microsuite V software. Results are mean \pm SEM of 20 fields. NS, not significant. f ATP production was measured using the Luminescent ATP detection kit (Abcam). Data are mean \pm S.D. of three independent experiments. g After $30 \mathrm{~min}$ of incubation with $500 \mathrm{nM}$ TMRE, mitochondrial membrane potential was monitored in a fluorescent plate reader. Results are mean \pm S.D. of three independent experiments. h TMRE signal was detected by FACS analysis. i Mean fluorescent intensity (MFI) of TMRE was calculated by using the CellQuest software. Results are mean \pm S.D. of three independent experiments 

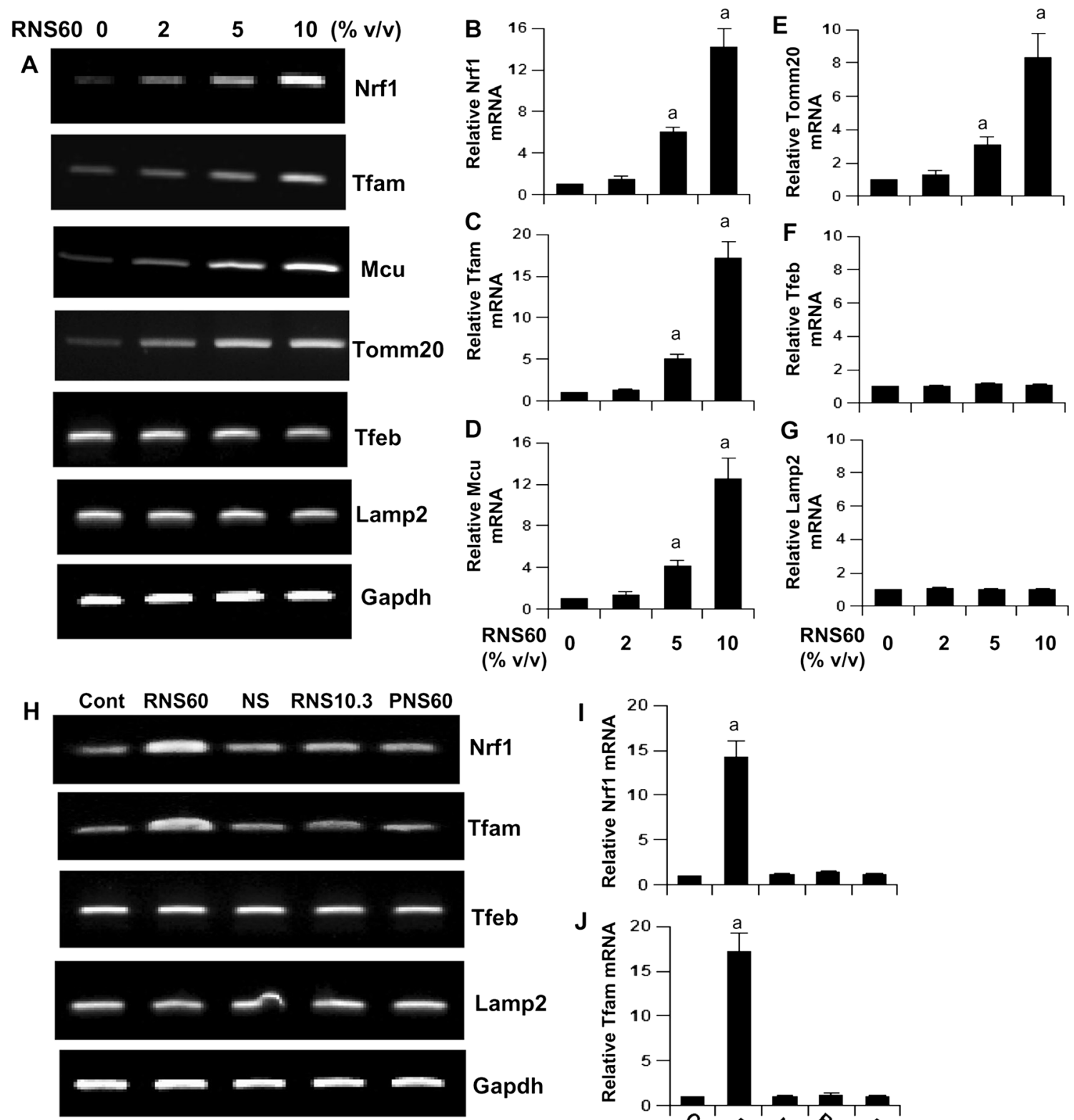

Tfeb

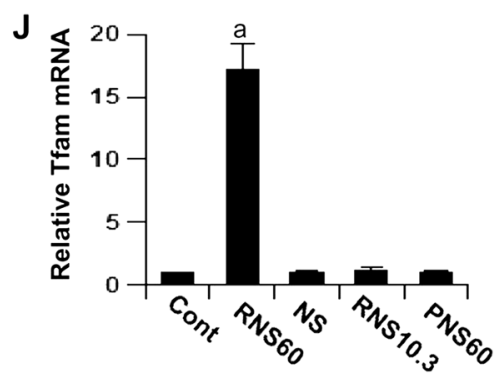

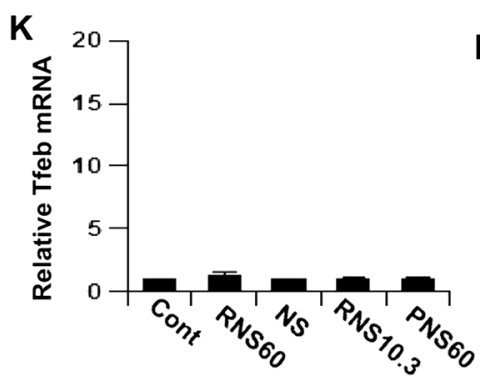

Fig. 3 RNS60 upregulates the expression of genes associated with mitochondrial biogenesis in MN9D mouse neuronal cells. Cells were treated with different concentrations of RNS60 for $5 \mathrm{~h}$ followed by monitoring the mRNA expression of Nrf1, Tfam, Mcu, Tomm20, Tfeb, and Lamp2 by RT-PCR (a) and real-time PCR (b, Nrfl; c, Tfam; d, Mcu; e, Tomm20; f, Tfeb; g, Lamp2). Results are mean \pm S.D. of three

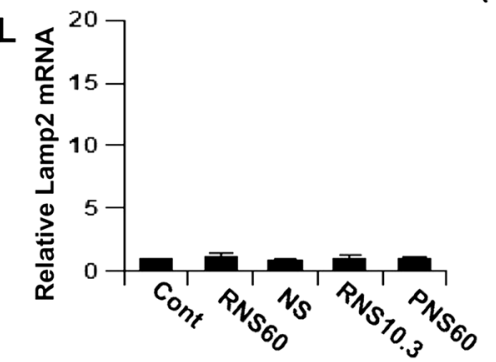

independent experiments. ${ }^{a} p<0.001$ versus control. Cells were treated with $10 \% v / v$ RNS60, NS, RNS10.3, and PNS60 for $5 \mathrm{~h}$ followed by monitoring the mRNA expression of Nrf1, Tfam, Tfeb, and Lamp2 by RT-PCR (h) and real-time PCR (i, Nrfl; j, Pgcla; k, Tfam; l, Tfeb). Results are mean \pm S.D. of three independent experiments. ${ }^{a} p<0.001$ versus control 

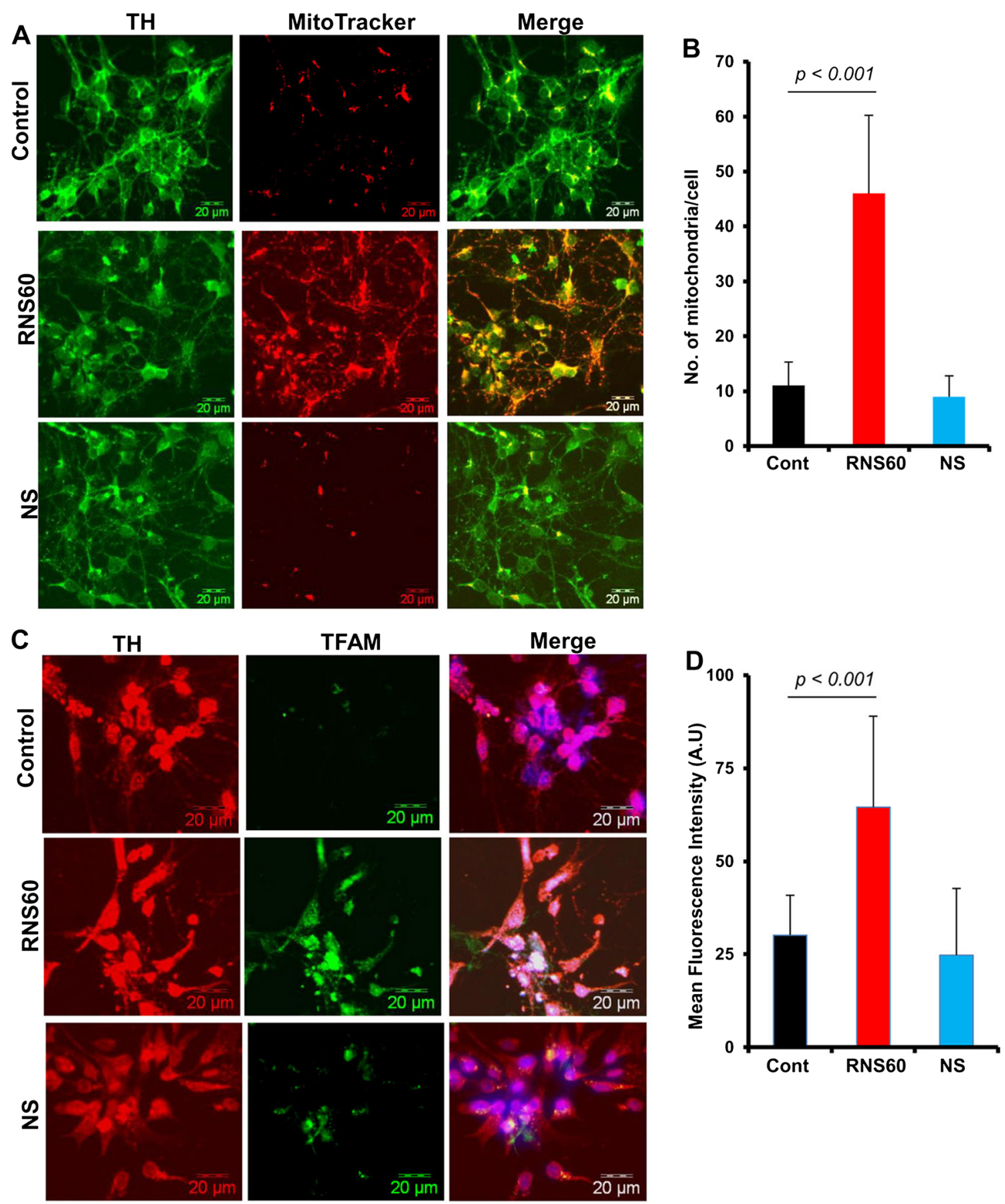

Fig. 4 RNS60 treatment upregulates mitochondrial biogenesis in mouse primary dopaminergic neurons. Neurons isolated from ventral mesencephalon of E12.5 to E14 d old fetus were treated with $10 \% \mathrm{v} / \mathrm{v}$ RNS60 or NS for $24 \mathrm{~h}$ followed by MitoTracker staining and immunostaining with anti-TH antibody (a). Number of mitochondria was counted in 25 different cells (b) and expressed as mitochondria per

cell. Data are mean \pm SEM of 25 different cells per group. c) Under similar treatment condition, cells were double-labeled for $\mathrm{TH}$ and TFAM. d) Mean Fluorescence Intensity of TFAM was measured in 10 different images using the measurement module of microsuite $\mathrm{V}$ Olympus software. Data are mean \pm SEM of 10 different images per group

RNS60 Increases Mitochondrial Biogenesis in Primary Mouse Dopaminergic Neurons Results seen in a transformed cell line are often not reflective of events in primary cells. Therefore, next, we investigated whether RNS60 was able to induce mitochondrial biogenesis in primary dopaminergic neurons. Cells treated with RNS60 and NS for $24 \mathrm{~h}$ under serum-free condition were doublelabeled for MitoTracker and TH. Similar to MN9D neuronal cells, RNS60, but not NS, increased MitoTracker labeling in dopaminergic neurons (Fig. 4a-b). Again, we 
A
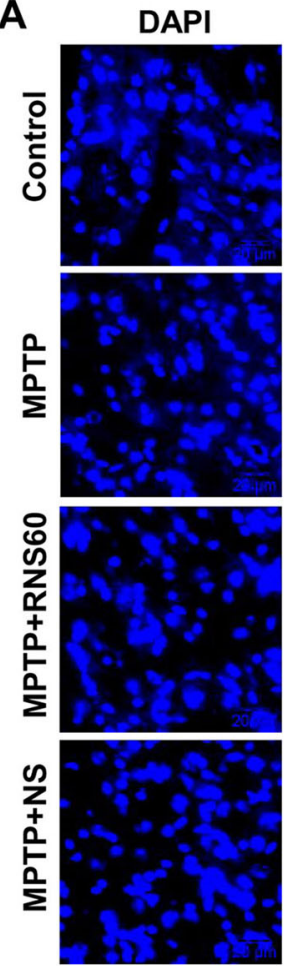

D
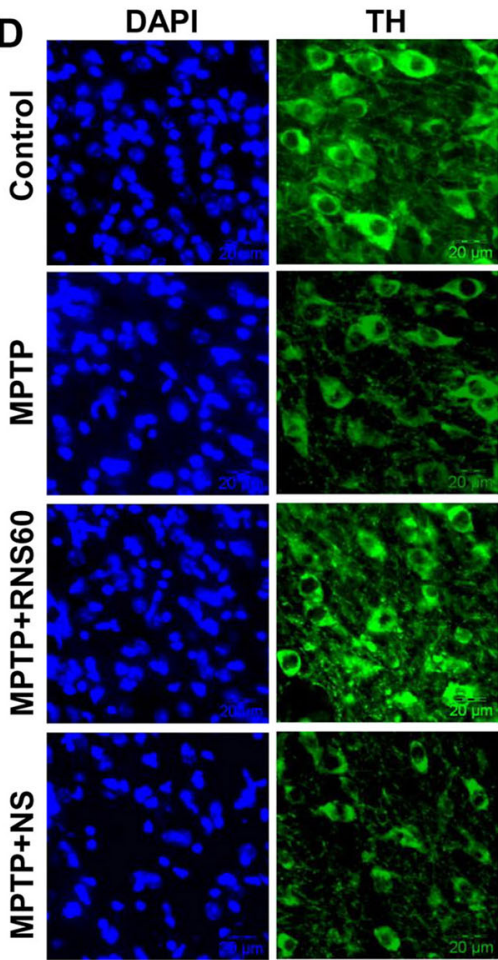

Fig. 5 RNS60 treatment upregulates mitochondrial biogenesis in vivo in the nigra of MPTP-intoxicated mice. Mice receiving either RNS60 or NS $(300 \mu \mathrm{l} / \mathrm{mouse} / \mathrm{d})$ via i.p. injection from $3 \mathrm{~h}$ after the last injection of MPTP were sacrificed 7 days after the last injection of MPTP followed by double-labeling of nigral sections with antibodies against
B
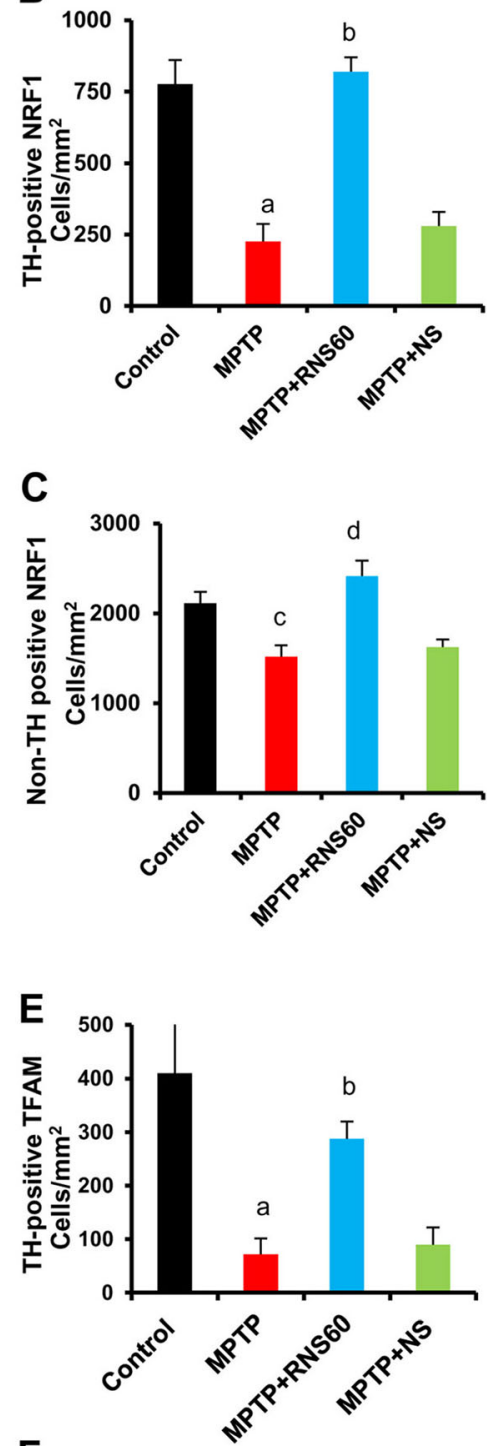

$F$

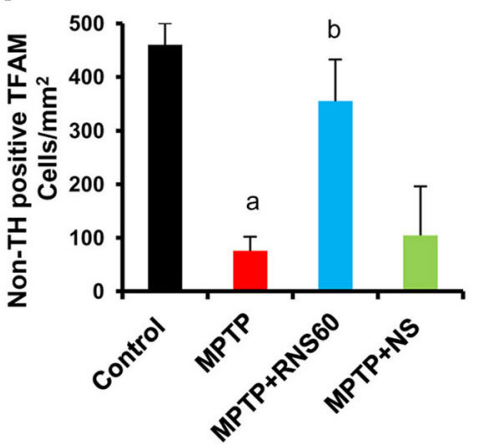

either TH \& NRF1 (a) or TH \& TFAM (d). Cells positive for NRF1 and TFAM (b, TH positive NRF1, c; Non TH positive NRF1; e, TH positive TFAM; f, Non TH positive TFAM) were counted in two nigral sections ( 2 images per slide) from each of five different mice. ${ }^{a} p<0.001$ versus control; ${ }^{b} p<0.001$ versus MPTP

cells were double-labeled for TFAM and TH after RNS60 treatment. In this case as well, RNS60, but not 


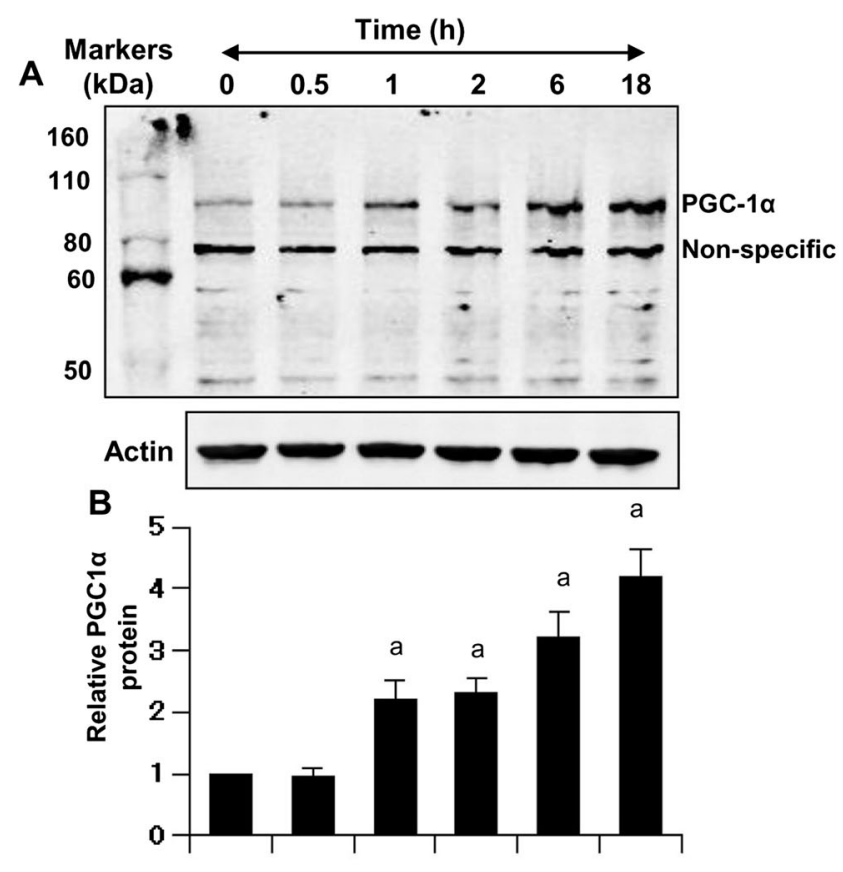

E
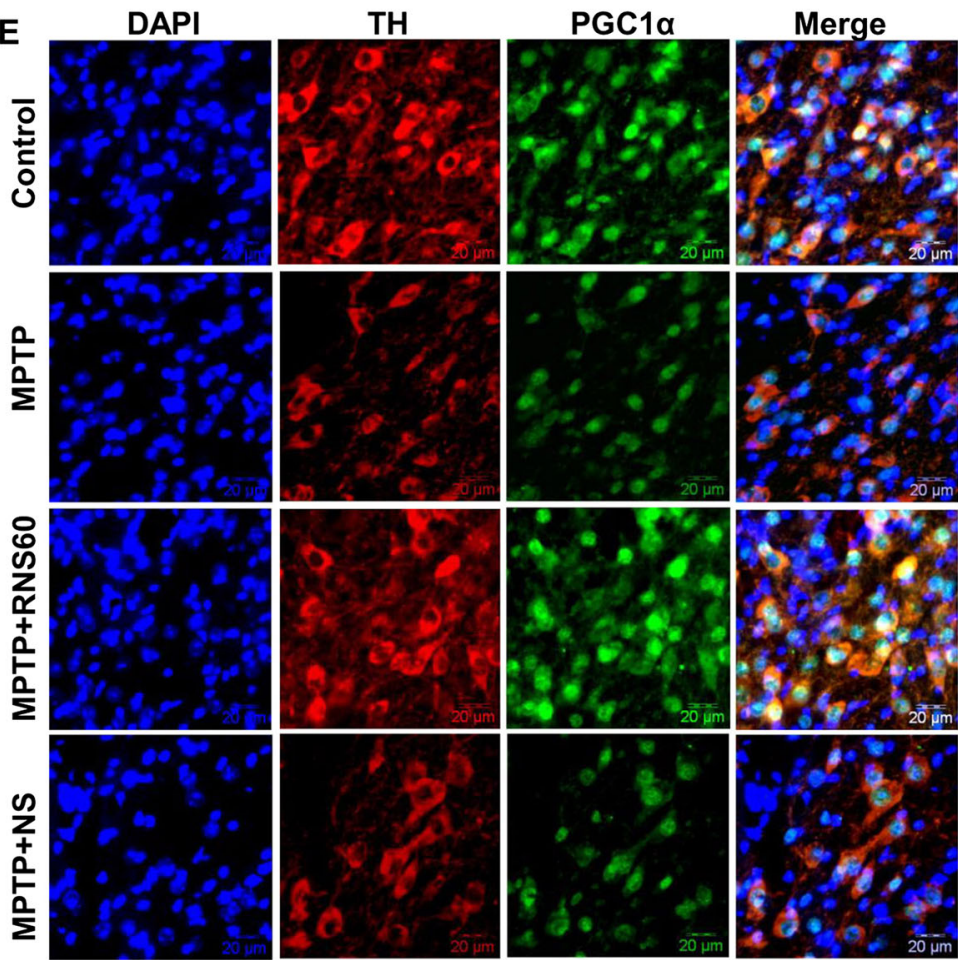

Fig. 6 Upregulation of PGC1 $\alpha$ by RNS60. MN9D cells were treated with $10 \% v / v$ RNS60 under serum-free condition for different time intervals followed by monitoring the level of PGC1 $\alpha$ by Western blot (a). Actin was run as loading control. Bands were scanned and values (PGC1 $\alpha /$ Actin) presented as relative to control (b). Results are mean \pm SD of three different experiments. ${ }^{a} p<0.05$ versus control; ${ }^{b} p<0.001$ versus control. MN9D cells were treated with different concentrations of RNS60 for $2 \mathrm{~h}$ followed by monitoring the mRNA expression of Pgcla by RT-PCR (c) and real-time PCR (d). Mice receiving either

NS, increased the level of TFAM in dopaminergic neurons (Fig. 4c-d). These results suggest that RNS60 is
C

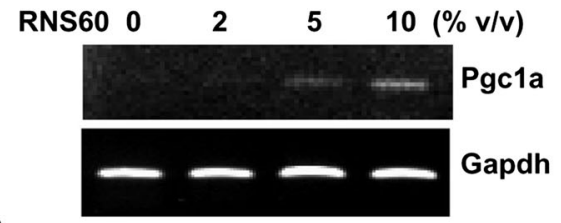

D
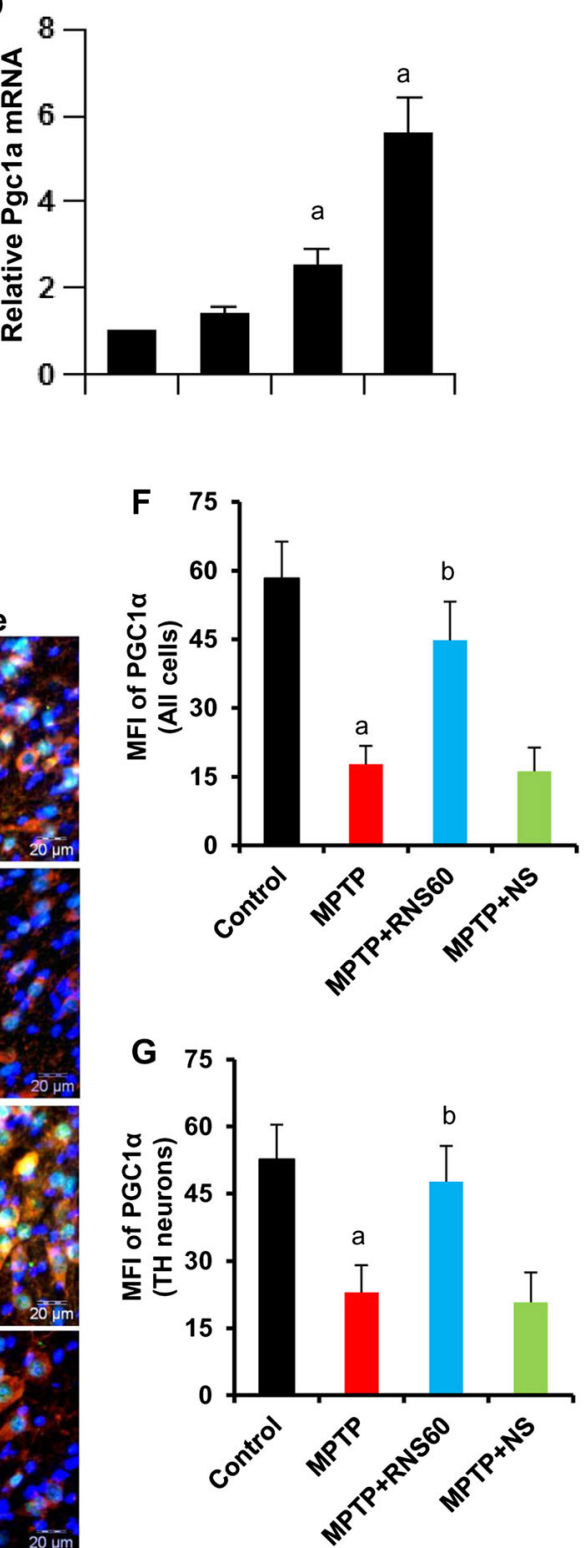

RNS60 or NS (300 $\mu \mathrm{l} / \mathrm{mouse} / \mathrm{d})$ via i.p. injection from $3 \mathrm{~h}$ after the last injection of MPTP were sacrificed 7 days after the last injection of MPTP followed by double-labeling of nigral sections with antibodies against $\mathrm{TH}$ $\& \mathrm{PGC} 1 \alpha(\mathbf{e})$. Mean fluorescence intensity (MFI) of PGC1 $\alpha$ in all cells (f) and TH-positive neurons (g) were calculated in two nigral sections (10 cells per section) from each of five different mice using the "measurement module" of Olympus Microsuite V software. ${ }^{a} p<0.001$ versus control; ${ }^{b} p<0.001$ versus MPTP

capable of stimulating mitochondrial biogenesis in primary dopaminergic neurons. 
RNS60 Treatment Protects and/or Stimulates Mitochondrial Biogenesis in the Nigra of MPTP-Intoxicated Mice Due to the lack of a proper detection tool to detect nanobubble-based structures, at present, we do not have any direct way to measure RNS60 within the CNS. However, within $3 \mathrm{~h}$ of intraperitoneal administration of RNS60, but not NS, we have observed the activation of class IA PI3K and the upregulation of $\mathrm{I} \kappa \mathrm{B} \alpha$, signature events of RNS60 (Khasnavis et al. 2012), in vivo in the nigra (Khasnavis et al. 2014). Accordingly, intraperitoneal administration of RNS60, but not NS, also protects dopaminergic neurons in MPTP mouse model of PD (Khasnavis et al. 2014). Therefore, it is likely that RNS60 enters the brain and we examined if RNS60 could modulate mitochondrial biogenesis in the nigra of MPTP-intoxicated mice. Mice received either RNS60 or NS $(300 \mu \mathrm{l} / \mathrm{mouse} / \mathrm{d})$ via intraperitoneal (i.p.) injection from $3 \mathrm{~h}$ after the last injection of MPTP and after 7 days of MPTP insult, mitochondrial biogenesis was monitored in nigral sections by doublelabeling of TH \& NRF1 (Fig. 5a-b) and TH \& TFAM (Fig. 5c-d). In control nigra, both NRF1 and TFAM were present in $\mathrm{TH}$-positive neurons as well as $\mathrm{TH}$ negative cells (Fig. 5). MPTP intoxication led to a decrease in mitochondrial content in the nigra as compared to control, which is evident from decrease in NRF1 (Fig. 5a-b) and TFAM (Fig. 5c-d) in the nigra. However, RNS60, but not NS, treatment led to the restoration of both NRF1 (Fig. 5a-b) and TFAM (Fig. 5cd) in the nigra of MPTP-insulted mice.

Upregulation of PGC1a in Dopaminergic Neurons by RNS60 Since PPAR $\gamma$ coactivator $1 \alpha(\mathrm{PGC} 1 \alpha)$ plays an important role in the biogenesis of mitochondria (Wareski et al. 2009), in order to understand the mechanisms behind RNS60mediated stimulation of mitochondrial biogenesis, we examined the effect of RNS60 on PGC1 $\alpha$. RNS60, but not NS, treatment rapidly increased the expression of $\mathrm{PGC} 1 \alpha$ protein in MN9D neuronal cells, which was evident as early as $2 \mathrm{~h}$ after RNS60 stimulation (Fig. 6a-b). The increase in PGC1 $\alpha$ mRNA by RNS60 (Fig. 6c-d) suggests that the upregulation occurs at the transcriptional level. Next we examined the effect of RNS60 on the level of PGC1 $\alpha$ in vivo in the nigra of MPTP-insulted mice. Although MPTP intoxication led to a decrease in $\mathrm{PGC} 1 \alpha$ in the nigra compared to control, RNS60 treatment resulted in the restoration and/or upregulation of nigral PGC $1 \alpha$ (Fig. 6e-f). This effect was specific as NS treatment had no such effect (Fig. 6e-f).

RNS60 Increases Mitochondrial Biogenesis in MN9D Dopaminergic Neuronal Cells via PGC1a Since RNS60 induced the level of $\mathrm{PGC} 1 \alpha$, we examined if induction of PGC1 $\alpha$ was essential for the upregulation of mitochondrial biogenesis by RNS60. We employed PGC $1 \propto$ siRNA to knockdown PGC $1 \alpha$ in neurons. As expected, PGC $1 \alpha$ siRNA, but not control siRNA, suppressed RNS60-mediated increase in PGC $1 \alpha$ protein in MN9D cells (Fig. 7a-b). Moreover, siRNA knockdown of PGC $1 \alpha$ inhibited RNS60mediated increase in Tfam, Nrfl, Mcu, and Tomm 20 mRNAs in MN9D neuronal cells (Fig. 7c). These results were confirmed by real-time PCR (Fig. 7d-g). Finally, we also monitored mitochondrial biogenesis. As expected, RNS60 increased MitoTracker labeling in MN9D neuronal cells (Fig. 7g). However, PGC1 $\alpha$ siRNA, but not control siRNA, markedly inhibited RNS60-mediated upregulation in MitoTracker labeling (Fig. 7g). These results demonstrate that RNS60 stimulates mitochondrial biogenesis in neuronal cells via PGC1 $\alpha$.

RNS60 Stimulates the Transcription of Pgc1a in MN9D Dopaminergic Neuronal Cells via Class IA PI3K-Mediated Activation of CREB Next, we investigated mechanisms by which RNS60 upregulated PGC1 $\alpha$. Earlier we have delineated that RNS60 rapidly activates phosphatidylinositol-3 kinase (PI3K) in microglial cells (Khasnavis et al. 2012). Because $\mathrm{PI} 3 \mathrm{~K}$ is associated to multiple cellular functions, we examined if PI3K was involved in RNS60-mediated increase in PGC1 $\alpha$ in MN9D neuronal cells. At first, we tested the effect of RNS60 on PI3K activation. Class IA PI3K, which is regulated by multiple receptor tyrosine kinases, consists of a heterodimer of a regulatory $85-\mathrm{kDa}$ subunit and a catalytic $110-\mathrm{kDa}$ subunit (p85:p110 $\alpha / \beta$ ). On the other hand, class IB PI3K consists of a dimer of a $101-\mathrm{kDa}$ regulatory subunit and a

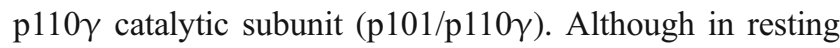
condition, PI3K subunits are located mainly in cytoplasm, upon activation, these are translocated to the plasma membrane (Franke et al. 1997; Koyasu 2003). Therefore, we monitored the activation of class IA and IB PI3K by the recruitment of $\mathrm{p} 110 \alpha, \mathrm{p} 110 \beta$ and $\mathrm{p} 110 \gamma$ to the plasma membrane. Western blotting of membrane fractions for p110 subunits showed that RNS60 specifically induced the recruitment of $\mathrm{p} 110 \alpha$ and $\mathrm{p} 110 \beta$, but not $\mathrm{p} 110 \gamma$, to the plasma membrane (Fig. 8a). Densitometric analysis of the p110 $\alpha$ and $\mathrm{p} 110 \beta$ at different time points of RNS60 stimulation indicates significant activation of $\mathrm{p} 110 \alpha \mathrm{PI} \mathrm{K}$ at 10 and $15 \mathrm{~min}$ and $\mathrm{p} 110 \beta$ PI3K at 5, 10 and $15 \mathrm{~min}$ (Fig. 8b). Again these results were specific as NS remained unable to activate $\mathrm{p} 110 \alpha$ and $\mathrm{p} 110 \beta$ PI3K at any time points tested. Together, these results suggest that RNS60 activates class IA PI3K p110 $\alpha$ and p110 $\beta$, but not class IB PI3K p110 $\gamma$, in MN9D neuronal cells.

Next, to delineate whether PI3K is involved in RNS60mediated upregulation of PGC $1 \alpha$, cells were pretreated with different concentrations of LY294002, a morpholinecontaining chemical compound that strongly inhibits PI3K, for $30 \mathrm{~min}$ followed by stimulation with $10 \%$ RNS60 for $2 \mathrm{~h}$ and analysis of Pgcla mRNA. As expected, RNS60 treatment increased the mRNA expression of Pgcla (Fig. 8c-d). 

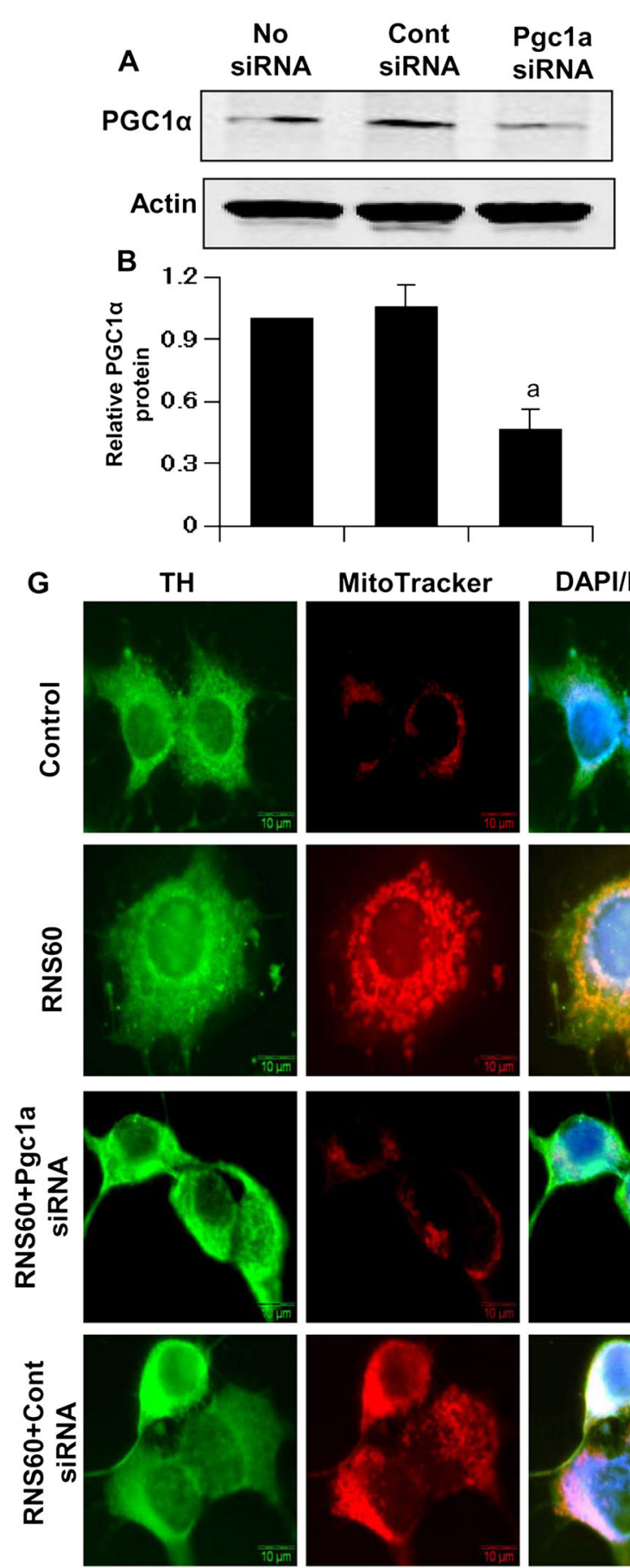

Fig. 7 SiRNA knockdown of PGC1 $\alpha$ abrogates RNS60-mediated mitochondrial biogenesis in MN9D mouse neuronal cells. Cells were transfected with control or PGC1 $\alpha$ siRNAs. After $48 \mathrm{~h}$ of transfection, cells were treated with $10 \% v / v$ RNS60 for $4 \mathrm{~h}$ followed by monitoring the level of PGC1 $\alpha$ by Western blot (a). Actin was run as loading control. Bands were scanned and values (PGC1 $\alpha /$ Actin) presented as relative to control (b). Results are mean $\pm \mathrm{SD}$ of three different experiments. ${ }^{a} p<0.001$ versus control; ${ }^{b} p<0.001$ versus control siRNA-RNS60.

However, pretreatment with LY294002 abrogated RNS60mediated increase in Pgcla expression in neuronal cells (Fig. $8 \mathrm{c}-$ d). To further confirm, we performed TH:PGC1 $\alpha$

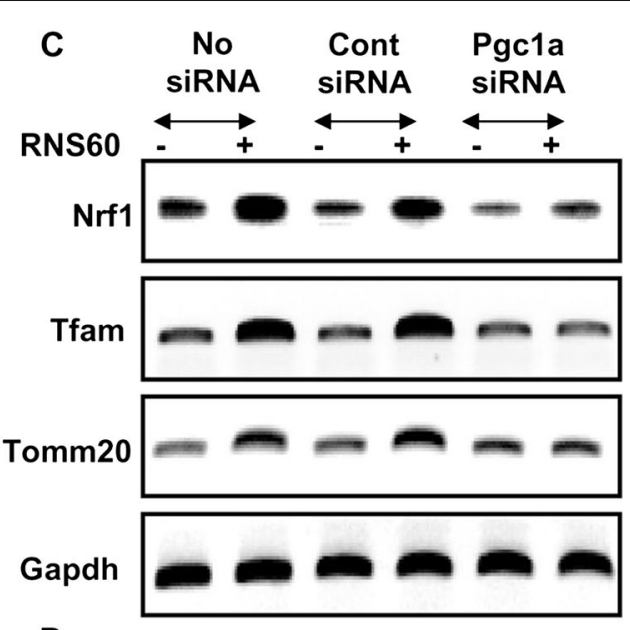

D
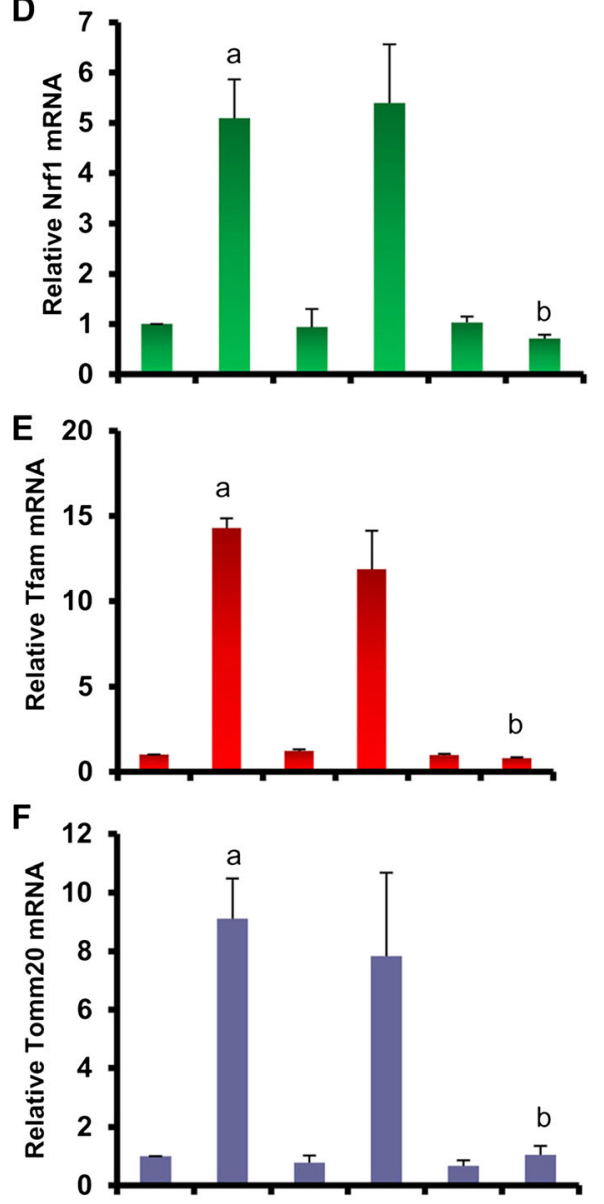

After $48 \mathrm{~h}$ of transfection, cells were treated with $10 \% v / v$ RNS60 for $5 \mathrm{~h}$ followed by monitoring the mRNA expression of Nrf1, Tfam and Tomm 20 by RT-PCR (c) and real-time PCR (d, Nrf1; e, Tfam; f, Tomm20). Results are mean $\pm \mathrm{SD}$ of three different experiments. ${ }^{a} p<$ 0.001 versus control; ${ }^{b} p<0.001$ versus RNS60. g) After $48 \mathrm{~h}$ of transfection, cells were treated with $10 \% \mathrm{v} / \mathrm{v}$ RNS60 for $24 \mathrm{~h}$ followed by double-labeling for MitoTracker and TH. Results represent three independent experiments double labeling. Although RNS60 did not modulate TH, marked increase in PGC1 $\alpha$ protein was seen after RNS60 treatment (Fig. 8e). However, LY294002 strongly suppressed 


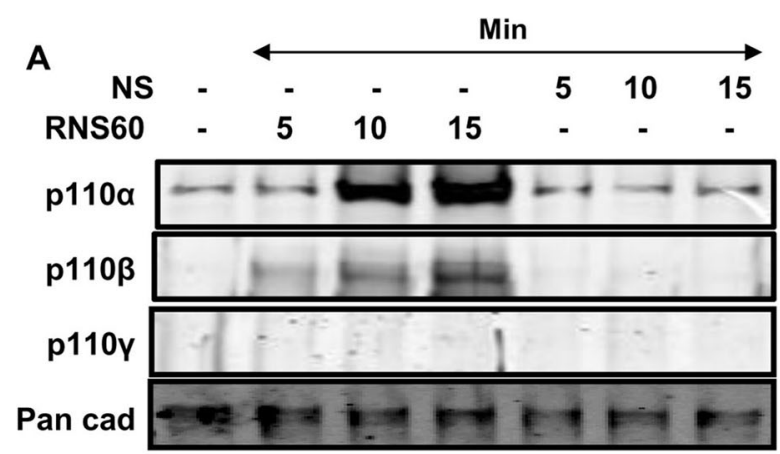

$\begin{array}{cccccc}\mathrm{RNS60} & - & + & + & + & - \\ \operatorname{LY}(\mu \mathrm{M}) & 0 & 0 & 2 & 5 & 5\end{array}$

C
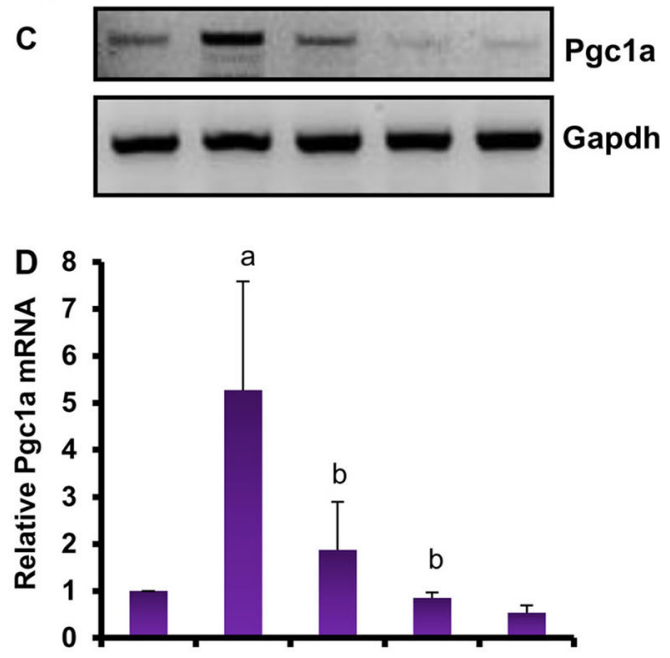

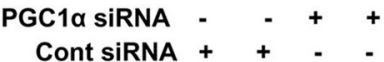

E
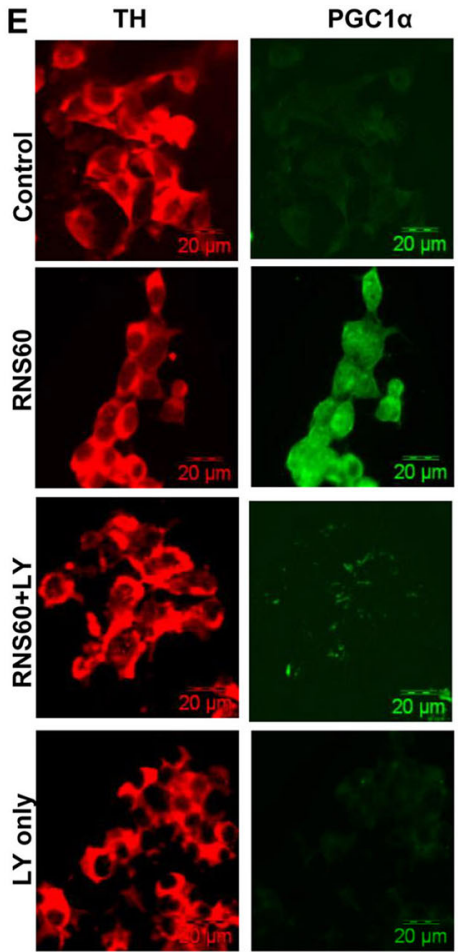

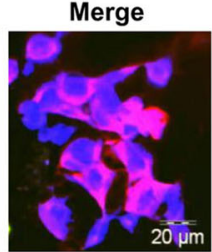

RNS60

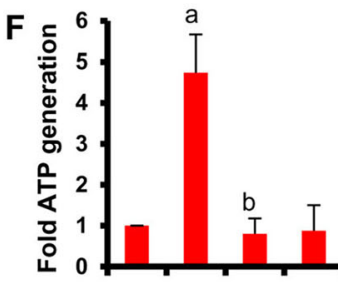

G
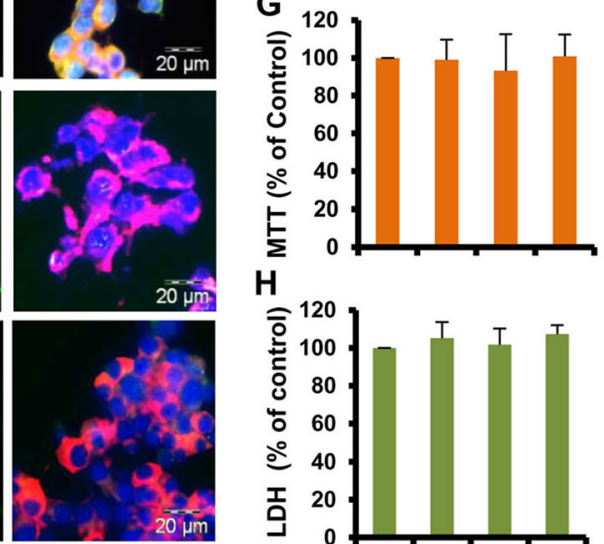

$\mathrm{H}$

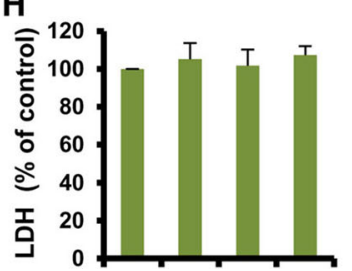

RNS60 - + - +

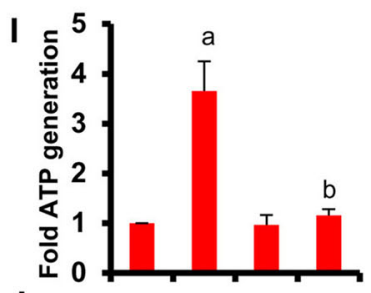

J
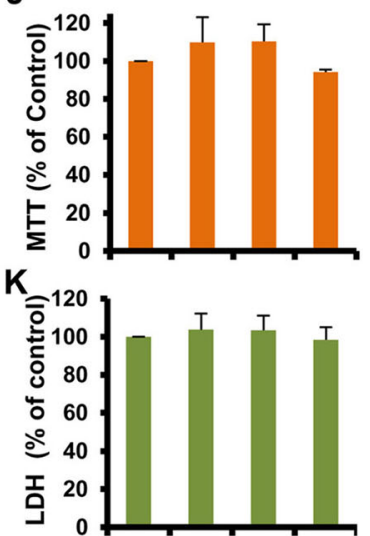

RNS60-mediated upregulation of PGC1 $\alpha$ (Fig. 8e). These results suggest that RNS60 upregulates $\mathrm{PGC} 1 \alpha$ in MN9D neuronal cells via PI3K.

Since RNS60 increased the production of ATP (Fig. 2d), we examined if RNS60 required the PI3K-PGC1 $\alpha$ pathway for stimulating ATP production in neurons. Suppression of RNS60-induced ATP production by LY294002 suggests the involvement of PI3K in RNS60-mediated ATP generation. Similarly, PGC1 $\alpha$ siRNA, but not control siRNA, also reduced the production of ATP in RNS60-stimulated neuronal cells, indicating the involvement of $\mathrm{PGC} 1 \alpha$ in this process. These results were not due to any change in viability as evident from MTT and LDH release assays (Fig. $8 \mathrm{~g}-\mathrm{h}$ and $\mathrm{j}-\mathrm{k}$ ). Although RNS60 protected neurons from A $\beta 1-42$-mediated 
Fig. 8 Role of PI3 kinase in RNS60-mediated upregulation of PGC1 $\alpha$ and ATP generation in MN9D mouse neuronal cells. a Cells were treated with $10 \% v / v$ RNS60 or NS for different minute intervals under serum-free condition followed by monitoring the levels of $\mathrm{p} 110 \alpha, \mathrm{p} 110 \beta$ and $\mathrm{p} 110 \gamma$ in cell membranes by Western blot. Pan cadherin was run as a membrane marker. b Bands were scanned and values (p110 $/$ pan Cad, p110 $\beta /$ pan Cad and p110 $/$ pan Cad) are expressed as relative to control. Results are mean $\pm \mathrm{SD}$ of three different experiments. ${ }^{a} p<0.001$ versus control. Cells treated with different concentrations of LY294002 (LY) for $30 \mathrm{~min}$ were stimulated with $10 \% \mathrm{v} / \mathrm{v}$ RNS60 for $2 \mathrm{~h}$ min followed by monitoring the mRNA expression of Pgcl $\alpha$ by RT-PCR (c) and real-time PCR (d). Results are mean \pm SD of three different experiments. ${ }^{a} p<0.001$ versus control; ${ }^{b} p<0.001$ versus RNS60. e) Cells treated with $5 \mu \mathrm{M}$ LY for 30 min were stimulated with $10 \% v / v$ RNS60 for $6 \mathrm{~h}$ followed by double-labeling of TH and PGC1 $\alpha$. Results represent three independent experiments. Cells pretreated with $5 \mu \mathrm{M} \mathrm{LY}$ for $30 \mathrm{~min}$ were stimulated with $10 \% v / v$ RNS60 for $24 \mathrm{~h}$ followed by monitoring ATP production (f), MTT (g) and LDH (h). Results are mean $\pm \mathrm{SD}$ of three different experiments. ${ }^{a} p<0.001$ versus control; ${ }^{b} p<0.001$ versus RNS60. Cells were transfected with PGC1 $\alpha$ siRNA and control siRNA. After $48 \mathrm{~h}$ of transfection, cells were stimulated with $10 \% v / v$ RNS60 for $24 \mathrm{~h}$ followed by monitoring ATP production (i), MTT (j) and LDH (k). Results are mean \pm SD of three different experiments. ${ }^{a} p<0.001$ versus untreated control siRNA; ${ }^{b} p<0.001$ versus control siRNA-RNS60

toxicity (Modi et al. 2014), RNS60 was unable to stimulate the survival of normal neurons (Fig. $8 \mathrm{~g}-\mathrm{h}$ and $\mathrm{j}-\mathrm{k}$ ).

Next, we investigated how PI3K coupled the transcription of Pgcla. While analyzing the Pgcla gene promoter by MatInspector V2.2 search machinery, we found a consensus cAMP response element (CRE) very close to the transcription start site (Fig. 9a). Since CREB can bind to CRE and we have previously found the involvement of class IA PI3K in the activation of CREB (Khasnavis et al. 2012), we examined if PI3K coupled the transcription of Pgcla via activation of CREB in RNS60-treated neuronal cells. At first, we monitored if RNS60 could induce the activation of CREB in MN9D neuronal cells. It is clearly evident from P-CREB and total CREB Western blots that RNS60, but not NS, markedly induced the phosphorylation of CREB within minute intervals without modulating the level of total CREB (Fig. 9b). These results were further confirmed by P-CREB (Fig. 9d) and total CREB (Fig. 9e) immunostaining of RNS60- and NStreated cells. Furthermore, consistent to our earlier finding in microglial cells (Khasnavis et al. 2012), here we have also observed that RNS60 induces the activation of CREB in MN9D neuronal cells via PI3K as RNS60mediated phosphorylation of CREB was dosedependently inhibited by LY294002 (Fig. 9f-g).

Next, by using ChIP analysis, we examined whether CREB was in fact recruited to the Pgcla promoter in MN9D neuronal cells after RNS60 treatment. Upon immunoprecipitation of chromatin fragments by antibodies against CREB, we were able to amplify 83-bp fragments flanking the CRE (Fig. 10a) in RNS60-treated, but neither NS-treated nor control untreated, neuronal cells (Fig. 10b-c). On the other hand, no amplification product was observed in any of the immunoprecipitates obtained with control IgG (Fig. 10b-c), suggesting the specificity of these interactions. Furthermore, immunoprecipitation of chromatin fragments by antibodies against CBP and p300 suggests that RNS60 induced the recruitment of CBP, but not p300, to the Pgcla gene promoter in MN9D neuronal cells (Fig. 10d-e). Consistent to the recruitment of CREB to the Pgcla promoter, siRNA knockdown of CREB (Fig. 11ab) suppressed RNS60-mediated upregulation of $\mathrm{Pgcla}$ mRNA (Fig. 11c-d) in MN9D neuronal cells. Accordingly, siRNA knockdown of CREB abrogated RNS60mediated upregulation of Nrf1 (Fig. 11e) and Tfam (Fig. 11f) and increase in mitochondrial biogenesis (Fig. $11 \mathrm{~g}-\mathrm{h}$ ). Together, these results demonstrate that RNS60 increases mitochondrial biogenesis in neuronal cells via class IA PI3K-CREB-mediated upregulation of $\operatorname{PGC} 1 \alpha$.

\section{Discussion}

RNS60 Stimulates Mitochondrial Biogenesis in Dopaminergic Neurons The mitochondria is the most important organelle in cells that not only participates in energy homeostasis and metabolism but also play significant roles in other biological processes like signal transduction, aging, oxidative stress, and apoptosis (Mizuno et al. 1995; Baloyannis 2006; Zsurka and Kunz 2015). Accordingly, the abundance, morphology, and functional properties of mitochondria are finely tuned to meet cell-specific energetic, metabolic, and signaling demands. As a result, decreased mitochondrial biogenesis and function are seen in many pathological conditions including neurodegenerative disorders (Dong et al. 2015; Wang et al. 2016). Therefore, the ability to manipulate or influence mitochondrial biogenesis in neurons may be of therapeutic importance for neurodegenerative diseases. Although there may be other approaches to stimulate mitochondrial biogenesis, here we introduce a simple saline-based agent to achieve this. RNS60 is an electrokinetically modified saline that contains charge-stabilized nanobubbles, but no active pharmaceutical ingredients. Due to Taylor-Couette-Poiseuille (TCP) turbulence, RNS60 is proposed to contain charge-stabilized nanostructures consisting of an oxygen nanobubble core surrounded by an electrical double-layer at the liquid/ gas interface (Khasnavis et al. 2012). Several lines of evidence presented in this manuscript clearly demonstrate that RNS60 is capable of upregulating mitochondrial biogenesis in dopaminergic neurons. Our 

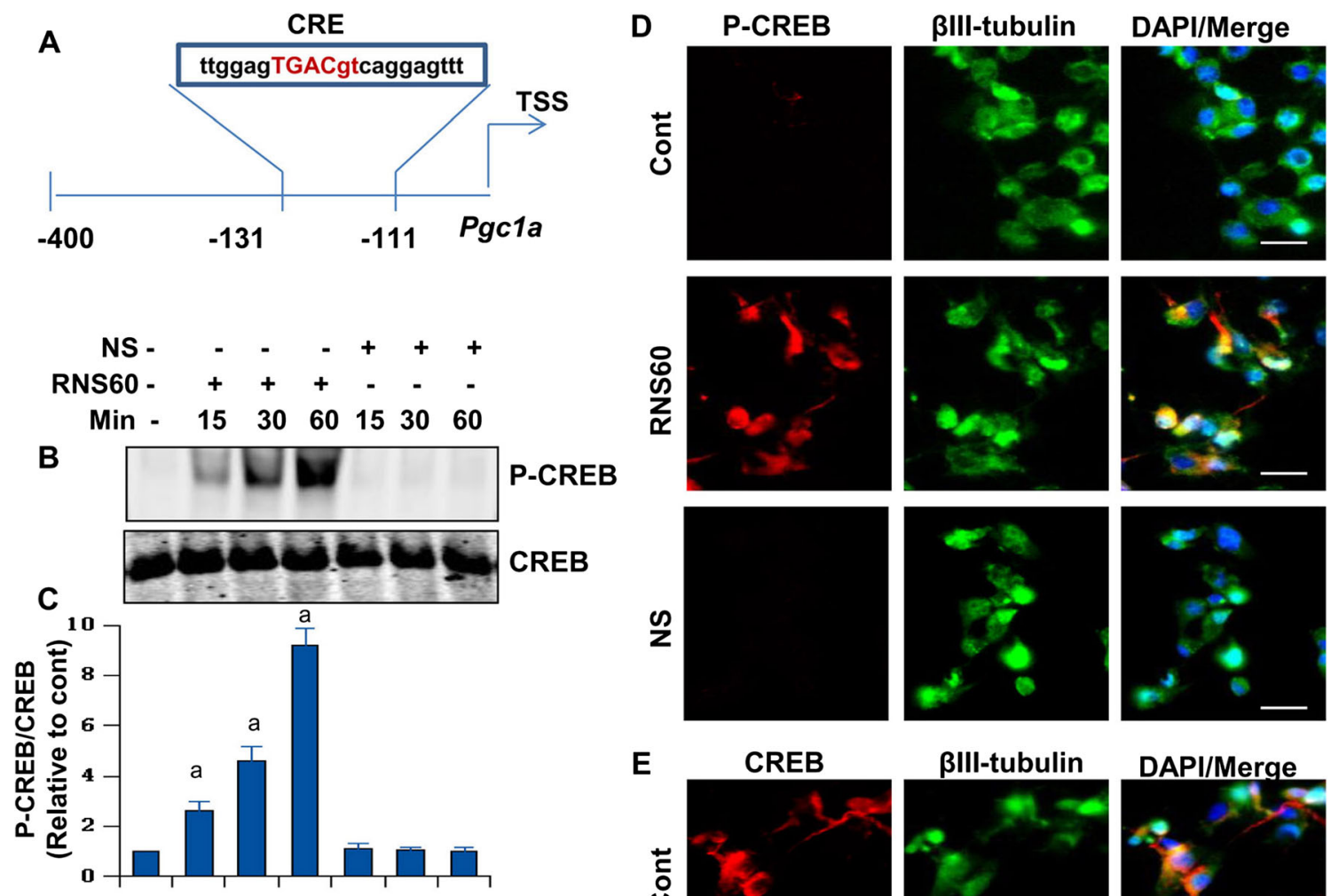

P-CREB

CREB

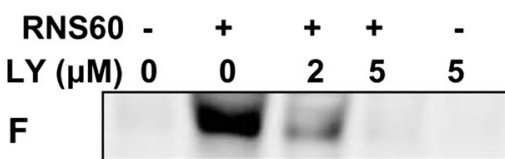

P-CREB

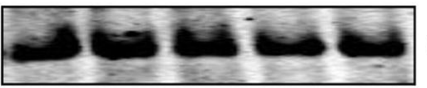
CREB
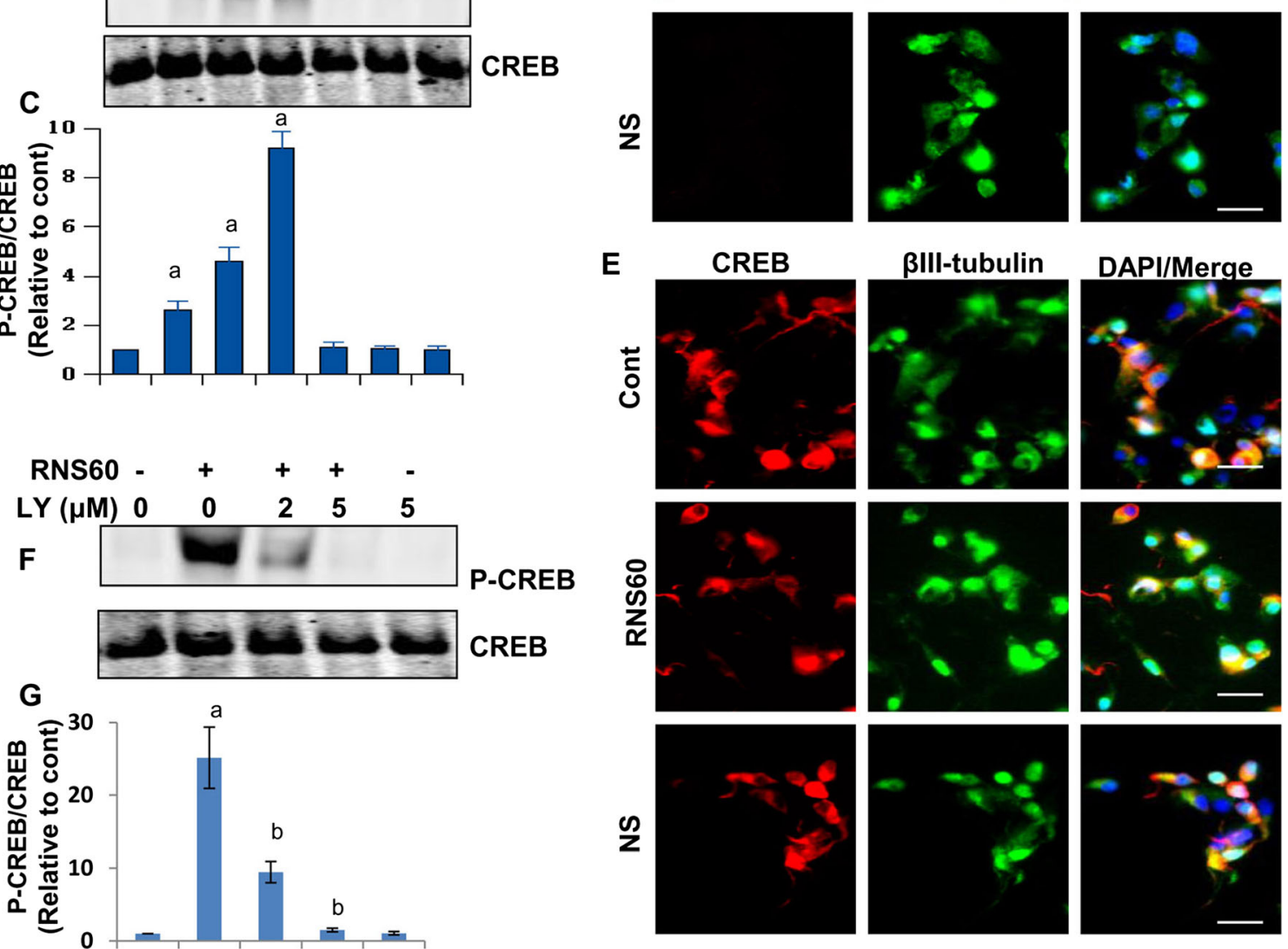

Fig. 9 Activation of CREB in MN9D mouse neuronal cells by RNS60 via PI3K. a Pgcl $\alpha$ gene promoter analysis shows the presence of a consensus cAMP response element (CRE) near the transcription start site (TSS). b Cells were treated with $10 \% v / v$ RNS60 or NS for different minute intervals under serum-free condition followed by monitoring the levels of phospho (P)-CREB and total (t) CREB by Western blot. c) Bands were scanned and values of (P-CREB/tCREB) are expressed as relative to control. Results are mean $\pm \mathrm{SD}$ of three different experiments. ${ }^{a} p<0.001$ versus control. After 60 min of treatment, cells were double-

labeled with either P-CREB \& $\beta$-tubulin (d) or P-CREB \& $\beta$-tubulin (e). Results represent three independent experiments. $\mathbf{f}$ Cells treated with different concentrations of LY294002 for 30 min were stimulated with $10 \% v / v$ RNS60 for 60 min followed by monitoring the levels of P-CREB and tCREB by Western blot. $\mathrm{g}$ Bands were scanned and values of (P$\mathrm{CREB} / \mathrm{tCREB}$ ) are expressed as relative to control. Results are mean \pm SD of three different experiments. ${ }^{a} p<0.001$ versus control; ${ }^{b} p<0.001$ versus RNS60

conclusion is based on the following findings. First, we observed that RNS60, but not NS, RNA10.3 or PNS60, stimulated mitochondrial biogenesis in MN9D dopaminergic neuronal cells. Interestingly, RNS60 did not increase lysosomal biogenesis in MN9D cells. Second, RNS60, but not NS, RNS10.3 or PNS60, upregulated

the expression of Nrfl, Tfam, Mcu, and Tom20, genes associated with mitochondrial biogenesis, in MN9D cells. However, RNS60 did not modulate the expression of $T f e b$ and Lamp2, genes associated with lysosomal biogenesis. Third, electron microscopy also revealed increase in mitochondria, but not lysosomes, by RNS60 in 
Fig. 10 RNS60 induces the recruitment of CREB to the Pgc1a promoter in MN9D neuronal cells. a Position of the Pgcla promoter that was targeted during ChIP analysis. Cells were treated with $10 \% v / v$ RNS60 or $\mathrm{NS}$ for $2 \mathrm{~h}$ and the recruitment of CREB to the Pgcl $\alpha$ promoter was monitored by ChIP followed by PCR (b) and real-time PCR (c). Values (CREB Ab IP/IgG IP) are expressed as relative to control. Results are mean $\pm \mathrm{SD}$ of three different experiments. ${ }^{a} p<$ 0.001 versus control. The recruitment of CBP and p300 to the $P g c 1 \alpha$ promoter was monitored by ChIP followed by PCR (d) and real-time PCR (e). Values (CBP or p300 Ab IP/IgG IP) are expressed as relative to control. Results are mean \pm SD of three different experiments. ${ }^{a} p<0.001$ versus control
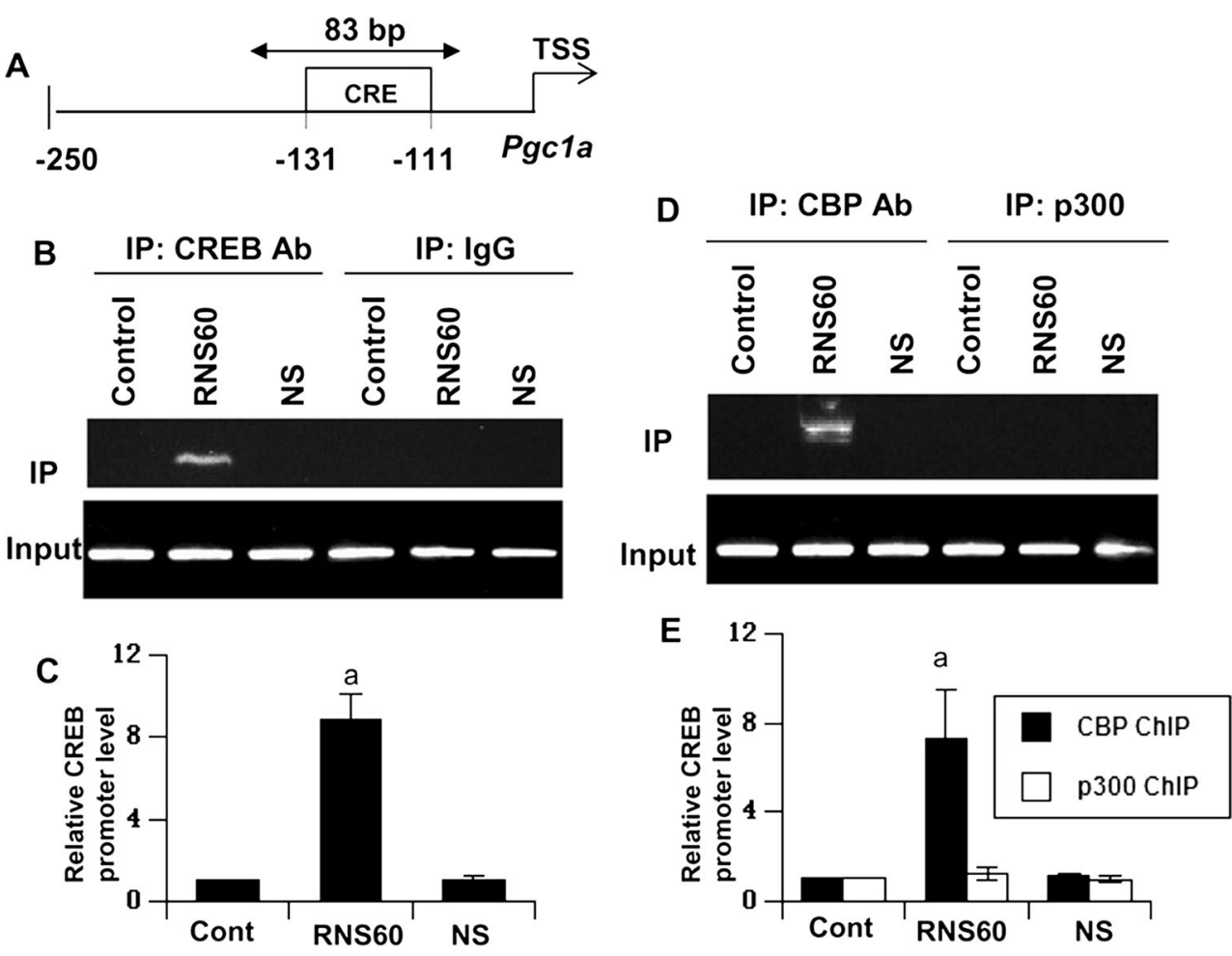

MN9D cells. Fourth, RNS60 also increased mitochondrial biogenesis in primary mouse dopaminergic neurons. Fourth, while the level of TFAM decreased in the nigra of MPTP mouse model of PD, RNS60 treatment upregulated and/or protected nigral TFAM from MPTP toxicity. Recently we have delineated protection of dopaminergic neurons and restoration of locomotor activities in MPTP-intoxicated mice by RNS60 (Khasnavis et al. 2014). Since mitochondrial dysfunction is known to contribute to nigrostriatal pathology in PD patients and in animal models of PD (Mizuno et al. 1995; Surmeier and Sulzer 2013), our current results suggest that this mitochondria-boosting effect of RNS60 may play a role in RNS60-mediated protection of the nigrostriatum in MPTP mouse model.

\section{RNS60 Requires PGC1a to Stimulate Mitochondrial Biogenesis} Mitochondrial biogenesis is a complex process that requires a coordinated regulation of synthesis, import, and incorporation of proteins and lipids to the existing mitochondrial reticulum, as well as replication of the mitochondrial DNA. A major breakthrough in our understanding of how mitochondrial biogenesis is coordinately regulated was the identification of NRF-1, NRF-2 and PPAR $\gamma$, transcription factors that are responsible for mitochondrial biogenesis. Accordingly, it has been found that PPAR $\gamma$ coactivator $1 \alpha(\operatorname{PGC} 1 \alpha)$, functioning as a coactivator of NRF-1, NRF-2 and PPAR $\gamma$, is capable of integrating various physiological signals and enhancing mitochondrial biogenesis ( Wu and Boss 2007; Wareski et al. 2009; Villena 2015). Accordingly, we observed upregulation of PGC $1 \alpha$ by RNS60 in MN9D neuronal cells. Peripheral administration of RNS60 also increased PGC1 $\alpha$ in THir neurons as well as other brain cells in vivo in the nigra of MPTP intoxicated mice. Suppression of Nrf1, Tfam, Mcu, and Tom 20 gene expression and attenuation of mitochondrial biogenesis in RNS60-stimulated neuronal cells by siRNA knockdown of PGC $1 \alpha$ suggests that RNS60 stimulates mitochondrial biogenesis via $\mathrm{PGC} 1 \alpha$.

\section{RNS60 Upregulates PGC1a in Neuronal Cells via Class IA PI3K-} Mediated Activation of CREB Mechanisms by which PGC1 $\alpha$ is upregulated are poorly understood. Recently we have delineated that RNS60 increases I $\mathrm{K} \mathrm{B} \alpha$ in microglia via the activation of class IA PI3K p $110 \alpha / \beta$, but not class IB PI3K p110 $\gamma$ (Khasnavis et al. 2012). PI3K is a key signaling molecule implicated in the regulation of a broad array of biological responses including metabolism, cell growth, cell survival, and apoptosis (Koyasu 2003). In case of class IA PI3K, the p85 regulatory subunit interacts with the IRS-1 through its $\mathrm{SH} 2$ domain and thus recruits the p110 catalytic subunit $(\mathrm{p} 110 \alpha / \beta)$ to the cell membrane, resulting in the activation of downstream signaling molecules like Akt/protein kinase B and p70 ribosomal S6 kinase (Koyasu 2003). While, for class IB PI3K, p110 $\gamma$ is activated by the engagement of G-protein coupled receptors. The $\mathrm{p} 110 \gamma$ then catalyzes the release of phosphatidylinositol $(3,4,5)$ - 

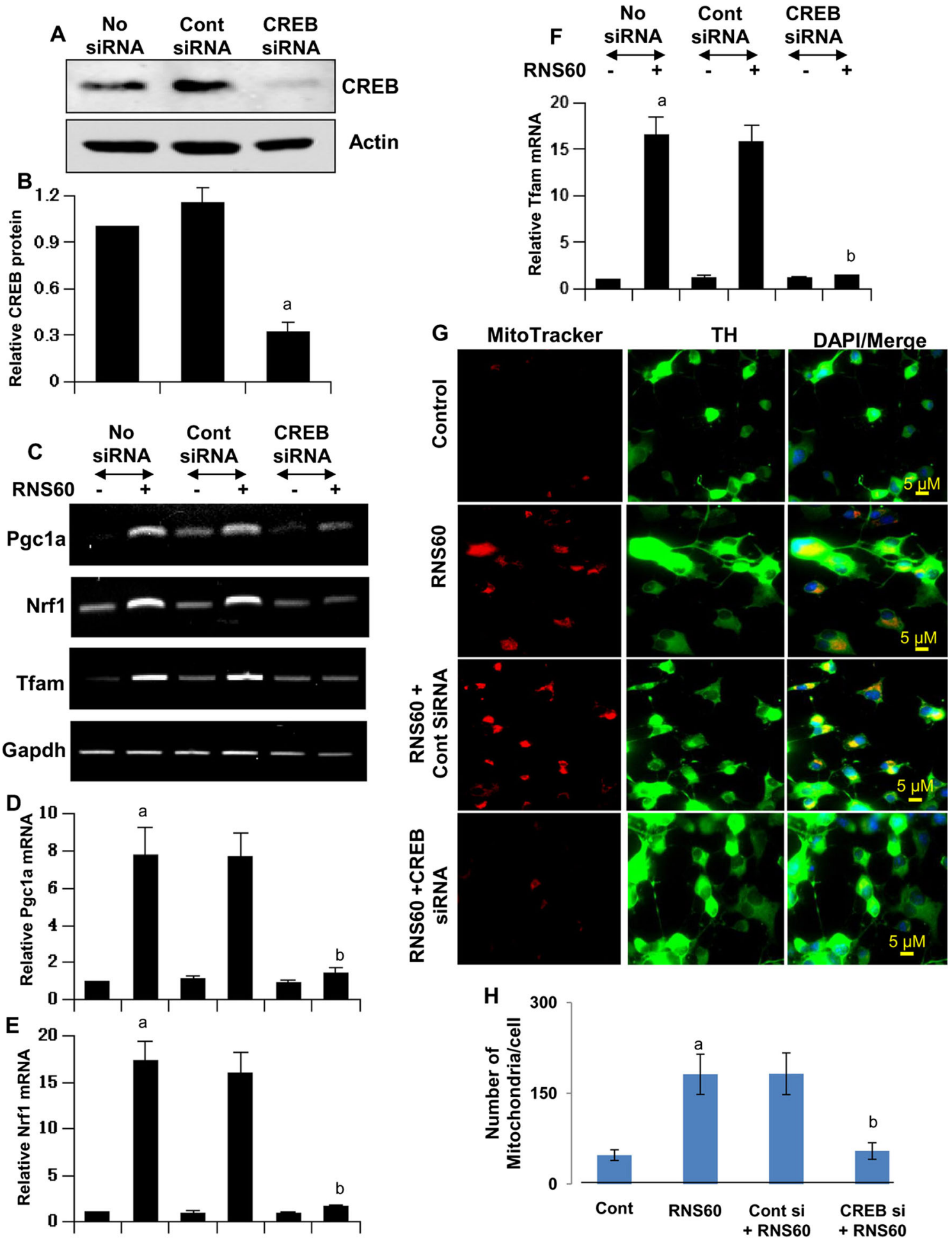

Fig. 11 SiRNA knockdown of CREB suppresses RNS60-mediated increase in PGC1 $\alpha$ and upregulation of mitochondrial biogenesis in MN9D neuronal cells. Cells were transfected with control or CREB siRNAs. After $48 \mathrm{~h}$ of transfection, the level of CREB was monitored by Western blot (a). Actin was run as loading control. Bands were scanned and values (CREB/Actin) presented as relative to control (b). Results are mean $\pm \mathrm{SD}$ of three different experiments. ${ }^{a} p<0.001$ versus control siRNA. After $48 \mathrm{~h}$ of siRNA transfection, cells were treated with $10 \% v / v$ RNS60 for $2 \mathrm{~h}$ followed by monitoring the mRNA expression of

$P g c l \alpha, N r f 1$ and Tfam by semi-quantitative RT-PCR (c) and real-time PCR (d, Pgcla; $\mathbf{e}$, Nrfl; f, Tfam). Results are mean \pm SD of three different experiments. ${ }^{a} p<0.001$ versus control; ${ }^{b} p<0.001$ versus control siRNARNS60. g) After $48 \mathrm{~h}$ of transfection, cells were treated with $10 \% \mathrm{v} / \mathrm{v}$ RNS60 for $24 \mathrm{~h}$ followed by double-labeling for MitoTracker and TH. h) Number of mitochondria was counted and expressed as mitochondria per cell. Data are mean \pm S.D. of three independent experiments. ${ }^{a} p<0.001$ versus control; ${ }^{b} p<0.001$ versus control siRNA-RNS60 
triphosphate as the second messenger from phosphatidylinositol (4,5)-bisphosphate and activates downstream signaling molecules (Franke et al. 1997). Here we demonstrate that RNS60 induces the activation of both the subunits of class IA PI3K (p110 $\alpha$ and $\mathrm{p} 110 \beta$ ) without modulating class IB PI3K p110 $\gamma$ in MN9D neurons, suggesting the specific activation of class IA $p 110 \alpha / \beta$ PI3K in dopaminergic neuronal cells. Earlier by using Atomic Force Microscopy, we have demonstrated that RNS60 contains nanobubbles and that nanobubble composition of RNS60 is different from other control saline solutions (Khasnavis et al. 2012). Nanobubbles may interact with cells based upon their charge and size as these charged entities possess the necessary energetics to affect the membrane. Consistent to the presence of activated PI3K at the membrane, RNS60 containing charge-stabilized nanobubbles induced the recruitment of PI3K subunits to the membrane of neuronal cells. Abrogation of RNS60-mediated increase in $\mathrm{PGC} 1 \alpha$ and upregulation of mitochondrial biogenesis in neuronal cells by inhibitors of PI3K suggest the involvement of PI3K in RNS60-mediated increase in mitochondrial biogenesis. Furthermore, we have seen that a consensus CRE is present in the Pgcla promoter near the transcription start site and that RNS60 induced the recruitment of CREB and CBP to this CRE in Pgcla promoter. Suppression of RNS60-induced CREB activation by inhibition of PI3K suggests that RNS60 induces the activation of CREB in neuronal cells via PI3K. Recently, we have delineated that Akt, the downstream mediator of PI3K, is responsible for the activation of CREB in RNS60-treated microglial cells (Khasnavis et al. 2012). Therefore, it is possible that a similar pathway exists in dopaminergic neurons as well. Furthermore, attenuation of RNS60-mediated increase in $\mathrm{PGC} 1 \alpha$ and mitochondrial biogenesis by siRNA knockdown of CREB suggests that RNS60 increases the transcription of Pgcla in neuronal cells via class IA PI3K-mediated activation of CREB, resulting in increased mitochondrial biogenesis.

In summary, we have demonstrated that RNS60 upregulates mitochondrial biogenesis in neuronal cells via class IA PI3K-CREB-mediated upregulation of PGC1 $\alpha$. These results highlight a novel mitochondria boosting property of RNS60 and suggest that this simple modified saline may be explored to rejuvenate degenerating neurons in PD and other neurodegenerative disorders where impaired mitochondrial function serves as a causative factor.

Acknowledgements This study was supported by Revalesio Corporation.

\section{Compliance with Ethical Standards}

Conflict of Interest None.
Open Access This article is distributed under the terms of the Creative Commons Attribution 4.0 International License (http:// creativecommons.org/licenses/by/4.0/), which permits unrestricted use, distribution, and reproduction in any medium, provided you give appropriate credit to the original author(s) and the source, provide a link to the Creative Commons license, and indicate if changes were made.

\section{References}

Albers DS, Beal MF (2002) Mitochondrial dysfunction in progressive supranuclear palsy. Neurochem Int 40:559-564

Baloyannis SJ (2006) Mitochondrial alterations in Alzheimer's disease. J Alzheimers Dis 9:119-126

Brahmachari S, Jana A, Pahan K (2009) Sodium benzoate, a metabolite of cinnamon and a food additive, reduces microglial and astroglial inflammatory responses. J Immunol 183:5917-5927

Carelli V, Rugolo M, Sgarbi G, Ghelli A, Zanna C, Baracca A, Lenaz G, Napoli E, Martinuzzi A, Solaini G (2004) Bioenergetics shapes cellular death pathways in Leber's hereditary optic neuropathy: a model of mitochondrial neurodegeneration. Biochim Biophys Acta 1658: 172-179

Choi HK, Won LA, Kontur PJ, Hammond DN, Fox AP, Wainer BH, Hoffmann PC, Heller A (1991) Immortalization of embryonic mesencephalic dopaminergic neurons by somatic cell fusion. Brain Res 552:67-76

Dasgupta S, Jana M, Zhou Y, Fung YK, Ghosh S, Pahan K (2004) Antineuroinflammatory effect of NF-kappaB essential modifierbinding domain peptides in the adoptive transfer model of experimental allergic encephalomyelitis. J Immunol 173:1344-1354

Dong Y, Heien ML, Maxson MM, Ewing AG (2008) Amperometric measurements of catecholamine release from single vesicles in MN9D cells. J Neurochem 107:1589-1595

Dong W, Wang F, Guo W, Zheng X, Chen Y, Zhang W, Shi H (2015) Abeta25-35 suppresses mitochondrial biogenesis in primary hippocampal neurons. Cell Mol Neurobiol 36:83-91

Fan W, Evans R (2015) PPARs and ERRs: molecular mediators of mitochondrial metabolism. Curr Opin Cell Biol 33:49-54

Franke TF, Kaplan DR, Cantley LC, Toker A (1997) Direct regulation of the Akt proto-oncogene product by phosphatidylinositol-3,4bisphosphate. Science 275:665-668

Ghosh A, Roy A, Liu X, Kordower JH, Mufson EJ, Hartley DM, Ghosh S, Mosley RL, Gendelman HE, Pahan K (2007) Selective inhibition of NF-kappaB activation prevents dopaminergic neuronal loss in a mouse model of Parkinson's disease. Proc Natl Acad Sci U S A 104: 18754-18759

Ghosh A, Roy A, Matras J, Brahmachari S, Gendelman HE, Pahan K (2009) Simvastatin inhibits the activation of $\mathrm{p} 21$ ras and prevents the loss of dopaminergic neurons in a mouse model of Parkinson's disease. J Neurosci 29:13543-13556

Ghosh A, Jana M, Modi K, Gonzalez FJ, Sims KB, Berry-Kravis E, Pahan K (2015) Activation of peroxisome proliferator-activated receptor alpha induces lysosomal biogenesis in brain cells: implications for lysosomal storage disorders. J Biol Chem 290:1030910324

Guedes-Dias P, Pinho BR, Soares TR, de Proenca J, Duchen MR, Oliveira JM (2016) Mitochondrial dynamics and quality control in Huntington's disease. Neurobiol Dis 90:51-57

Hirai K, Aliev G, Nunomura A, Fujioka H, Russell RL, Atwood CS, Johnson AB, Kress Y, Vinters HV, Tabaton M, Shimohama S, Cash AD, Siedlak SL, Harris PL, Jones PK, Petersen RB, Perry G, Smith MA (2001) Mitochondrial abnormalities in Alzheimer's disease. J Neurosci 21:3017-3023 
Jana M, Jana A, Liu X, Ghosh S, Pahan K (2007) Involvement of phosphatidylinositol 3-kinase-mediated up-regulation of I kappa B alpha in anti-inflammatory effect of gemfibrozil in microglia. J Immunol 179:4142-4152

Khasnavis S, Pahan K (2012) Sodium benzoate, a metabolite of cinnamon and a food additive, upregulates neuroprotective Parkinson disease protein DJ-1 in astrocytes and neurons. J NeuroImmune Pharmacol 7(2):424-435

Khasnavis S, Jana A, Roy A, Mazumder M, Bhushan B, Wood T, Ghosh S, Watson R, Pahan K (2012) Suppression of nuclear factor-kappaB activation and inflammation in microglia by physically modified saline. J Biol Chem 287:29529-29542

Khasnavis S, Roy A, Ghosh S, Watson R, Pahan K (2014) Protection of dopaminergic neurons in a mouse model of Parkinson's disease by a physically-modified saline containing charge-stabilized nanobubbles. J NeuroImmune Pharmacol 9:218-232

Koyasu S (2003) The role of PI3K in immune cells. Nat Immunol 4:313319

Kukat C, Larsson NG (2013) mtDNA makes a U-turn for the mitochondrial nucleoid. Trends Cell Biol 23:457-463

Mizuno Y, Ikebe S, Hattori N, Nakagawa-Hattori Y, Mochizuki H, Tanaka M, Ozawa T (1995) Role of mitochondria in the etiology and pathogenesis of Parkinson's disease. Biochim Biophys Acta 1271:265-274

Modi KK, Jana A, Ghosh S, Watson R, Pahan K (2014) A physicallymodified saline suppresses neuronal apoptosis, attenuates tau phosphorylation and protects memory in an animal model of Alzheimer's disease. PLoS One 9:e103606

Modi KK, Roy A, Brahmachari S, Rangasamy SB, Pahan K (2015) Cinnamon and its metabolite sodium benzoate attenuate the activation of $\mathrm{p} 21 \mathrm{rac}$ and protect memory and learning in an animal model of Alzheimer's disease. PLoS One 10:e0130398

Mondal S, Martinson JA, Ghosh S, Watson R, Pahan K (2012) Protection of Tregs, suppression of Th1 and Th17 cells, and amelioration of experimental allergic encephalomyelitis by a physically-modified saline. PLoS One 7:e51869

Perez RG, Waymire JC, Lin E, Liu JJ, Guo F, Zigmond MJ (2002) A role for alpha-synuclein in the regulation of dopamine biosynthesis. $\mathrm{J}$ Neurosci 22:3090-3099

Petrungaro C, Zimmermann KM, Kuttner V, Fischer M, Dengjel J, Bogeski I, Riemer J (2015) The ca(2+)-dependent release of the Mia40-induced MICU1-MICU2 Dimer from MCU regulates mitochondrial ca(2+) uptake. Cell Metab 22:721-733

Roy A, Pahan K (2013) Ankyrin repeat and BTB/POZ domain containing protein-2 inhibits the aggregation of alpha-synuclein: implications for Parkinson's disease. FEBS Lett 587:3567-3574
Roy A, Ghosh A, Jana A, Liu X, Brahmachari S, Gendelman HE, Pahan K (2012) Sodium phenylbutyrate controls neuroinflammatory and antioxidant activities and protects dopaminergic neurons in mouse models of Parkinson's disease. PLoS One 7:e38113

Roy A, Jana M, Corbett GT, Ramaswamy S, Kordower JH, Gonzalez FJ, Pahan K (2013) Regulation of cyclic AMP response element binding and hippocampal plasticity-related genes by peroxisome proliferator-activated receptor alpha. Cell Rep 4:724-737

Roy A, Modi KK, Khasnavis S, Ghosh S, Watson R, Pahan K (2014) Enhancement of morphological plasticity in hippocampal neurons by a physically modified saline via phosphatidylinositol-3 kinase. PLoS One 9:e101883

Roy A, Jana M, Kundu M, Corbett GT, Rangaswamy SB, Mishra RK, Luan CH, Gonzalez FJ, Pahan K (2015) HMG-CoA reductase inhibitors bind to PPARalpha to upregulate Neurotrophin expression in the brain and improve memory in mice. Cell Metab 22:253-265

Saha RN, Liu X, Pahan K (2006) Up-regulation of BDNF in astrocytes by TNF-alpha: a case for the neuroprotective role of cytokine. J NeuroImmune Pharmacol 1:212-222

Scarpulla RC (2002) Nuclear activators and coactivators in mammalian mitochondrial biogenesis. Biochim Biophys Acta 1576:1-14

Schleiff E, Turnbull JL (1998) Functional and structural properties of the mitochondrial outer membrane receptor Tom20. Biochemistry 37 : 13043-13051

Surmeier DJ, Sulzer D (2013) The pathology roadmap in Parkinson disease. Prion 7:85-91

Tafuri F, Ronchi D, Magri F, Comi GP, Corti S (2015) SOD1 misplacing and mitochondrial dysfunction in amyotrophic lateral sclerosis pathogenesis. Front Cell Neurosci 9:336

Villena JA (2015) New insights into PGC-1 coactivators: redefining their role in the regulation of mitochondrial function and beyond. FEBS J 282:647-672

Wang W, Wang X, Fujioka H, Hoppel C, Whone AL, Caldwell MA, Cullen PJ, Liu J, Zhu X (2016) Parkinson's disease-associated mutant VPS35 causes mitochondrial dysfunction by recycling DLP1 complexes. Nat Med 90:51-57

Wareski P, Vaarmann A, Choubey V, Safiulina D, Liiv J, Kuum M, Kaasik A (2009) PGC-1 \{alpha\} and PGC-1 \{beta\} regulate mitochondrial density in neurons. J Biol Chem 284:21379-21385

Wu Z, Boss O (2007) Targeting PGC-1 alpha to control energy homeostasis. Expert Opin Ther Targets 11:1329-1338

Zsurka G, Kunz WS (2015) Mitochondrial dysfunction and seizures: the neuronal energy crisis. Lancet Neurol 14:956-966 